HEAT TRANSFER - ASIAN RESEARCH (WILEY)

Accepted February $8^{\text {th }} 2020$

\title{
SIMULATION OF UNSTEADY NATURAL CONVECTION FLOW OF A CASSON VISCOPLASTIC FLUID IN A SQUARE ENCLOSURE UTILIZING A MAC ALGORITHM
}

\author{
T. S. Devi ${ }^{1}$, C V. Lakshmi ${ }^{1}$, K. Venkatadri ${ }^{2 *}$, V.R. Prasad ${ }^{3}$ and O. Anwar Bég ${ }^{4}$ \\ ${ }^{1}$ Department of Applied Mathematics, Sri Padmavathi Mahila University, Visva Vidyalayam, Tirupati 517502, AP, India. \\ ${ }^{2}$ Department of Mathematics, Sreenivasa Institute of Technology and Management Studies, Chittoor-517127, India. \\ ${ }^{3}$ Department of Mathematics, Vellore Institute of Technology, Vellore-517112, India. \\ ${ }^{4}$ Multiphysical Engineering Sciences Group, Aeronautical and Mechanical Engineering Division, University of Salford, \\ School of Science, Engineering and Environment (SEE), M5 4WT, UK. \\ *Corresponding Author: venkatadri.venki@gmail.com
}

\begin{abstract}
Non-Newtonian fluids are increasingly being deployed in energy systems and materials processing. Motivated by these developments, in the current study, a numerical simulation is performed on two-dimensional, unsteady buoyancy-driven flow in a square cavity filled with non-Newtonian fluid (Casson liquid). The enclosure geometry features vertical isothermal walls (with one at higher temperature than the other) and thermally insulated horizontal walls. The conservation equations for mass, momentum and energy are normalized via appropriate transformations and the resulting dimensionless partial differential boundary value problem is solved computationally with a Marker and Cell (MAC) algorithm which features a finite difference scheme along with a staggered grid system. The projection method is employed to evaluate the pressure term. Extensive visualizations of the impact of emerging physical parameters (Rayleigh number and Casson viscoplastic parameter) on streamline and isotherm distributions in the cavity are presented for fixed Prandtl number. Nusselt number i.e. heat transfer rate is increased with rising values of the Casson viscoplastic fluid parameter for any value of Rayleigh number. The density of streamlines increases with increasing values of Casson viscoplastic fluid parameter up to 1. Overall the Casson fluid parameter plays a vital role in controlling the convective heat transfer within the enclosure. The computations are relevant to hybrid solar collectors, materials fabrication (polymer melts) etc.
\end{abstract}

KEYWORDS: Free convection, square enclosure, unsteady flow, Non-Newtonian (Casson) fluid, finite difference scheme, Projection Method; isotherms; flow visualization.

\section{NOMENCLATURE}

g Gravitational acceleration

L Length of the square cavity

p Dimensional pressure

P Dimensionless pressure

Pr Prandtl number

$\mathrm{Ra}$ Rayleigh number 
T Temperature

$\theta \quad$ Dimensionless temperature

$\mathrm{u}, \mathrm{v} \quad$ Velocity components

U,V Dimensionless velocity components

$\mathrm{x}, \mathrm{y} \quad$ Cartesian co-ordinates

$\mathrm{X}, \mathrm{Y}$ Dimensionless Cartesian components

t Dimensional time

\section{Greek symbols}

$\rho_{f} \quad$ Density of Casson fluid

$\alpha \quad$ Thermal diffusivity

$\mu \quad$ Dynamic viscosity

$v \quad$ Kinematic viscosity

$\beta \quad$ Thermal expansion co-efficient

$\gamma \quad$ Casson rheological fluid parameter

$\tau \quad$ shear stress (and dimensionless time)

$\gamma \quad$ shear rate

$\pi=e_{i j} e_{i j}$

$e_{i j} \quad(\mathrm{i}, \mathrm{j})^{\mathrm{th}}$ component of the deformation rate

$\pi \quad$ the product of the component of deformation rate

$\pi_{c} \quad$ critical value

$\mu_{B} \quad$ plastic dynamic viscosity of the non-Newtonian fluid

$p_{y} \quad$ yield stress of the fluid.

\section{Subscripts}

c Cold

h Hot/Heat 


\section{INTRODUCTION}

Buoyancy-driven flow is an interesting flow phenomenon in heat transfer sciences and arises in diverse engineering systems including environmental comfort, thermal control of electronic components, geothermal and solar energy systems, materials fabrication etc. Several technological applications have featured increasingly non-Newtonian fluids since they offer certain advantages over Newtonian fluids in terms of viscosity, thermophysical characteristics and durability. This has motivated substantial interest in mathematical modelling of nonNewtonian heat transfer in different geometrical configurations including stretching sheet flows, wavy surfaces, inclined planes and within enclosures (cavities). Non-Newtonian (rheological) liquids are of many different and intriguing types including viscoplastic (where fluids require a yield stress to initiate flow), viscoelastic, micro-structural couple stress and micropolar fluids, among others. One model for viscoplastic liquids which has emerged as quite popular and relatively simple to implement is the Casson fluid model. This model adequately describes a variety of real liquids including jelly, honey, certain plastics and polymers. It is also applicable for modelling blood flow through vessels at high shear rates [1]. Casson's model has received significant interest in engineering science simulation and a variety of analytical and computational methods have been utilized in simulating Casson rheological behavior in energy and industrial systems. Zaib et al. [2] used a shooting numerical method to study the impact of viscous dissipation on the convective flow of Casson fluid towards a shrinking sheet. Prasad et al. [3] employed Keller's finite difference box scheme to simulate enrobing slip thermal boundary layer flows of a cylindrical body in Casson fluid. They noted that temperatures are decreased whereas skin friction is enhanced with greater Casson rheological parameter. Das et. al [4] deployed a Laplace transform technique to investigate the time-dependent double diffusive magnetic Casson fluid convective flow from a flat plate, observing that increasing Casson fluid parameter enhances concentration values whereas it decelerates the flow and reduces temperatures. Reddy et al. [5] used a Crank-Bicholson difference scheme and Bejan's methods of entropy generation minimization and heat line visualization to study the transient thermal convection Casson fluid flow external to a cylinder in a permeable medium. Ganganapalli et. al [6] used MATLAB shooting quadrature to analyze the influence of wall slip velocity, radiative flux and chemical reaction on Casson flow from a two-dimensional wedge, noting that increasing Casson fluid parameter enhances both velocity and temperature. Vasu et al. [7] implemented homotopy and generalized differential quadrature (GDQ) methods to simulate the impact of Casson viscoplasticity and bioconvection microorganism mass transfer in hydromagnetic rheological nanofluid flow from an extending sheet. Akbar et al. [8] studied electromagnetic propulsion of Casson liquids in ciliated tubes with power series methods.

Buoyancy driven convection in enclosures is an area of particular interest in solar collector design [9] and also semi-conductor or polymer melt synthesis [10]. Such two-dimensional flows usually require numerical methods to solve the nonlinear governing conservation equations. Most of the researchers to study the natural convection within the enclosure with air have a working fluid. In recent years, with the objective of improving the thermal performance of working fluids in enclosures, several investigators have studied a variety of 
non-Newtonian fluid models in enclosure convection. Alloui and Vasseur [11] derived semianalytical solutions for non-Newtonian convection in polymeric shear-thinning CarreauYasuda fluids in a vertical enclosure, observing the significant modification in heat transfer characteristics with pseudo-plastic rheological behavior. Kefayati [12] employed a Finite Difference Lattice Boltzmann method (FDLBM) to simulate the thermal convection in powerlaw fluids in a sinusoidal heated cavity showing that with increasing power-law rheological index (from pseudoplastic to dilatant) there is a suppression in heat transfer. Further studies of enclosure convection include Kefayati [13] (on magnetic power-law nanofluids), Jecl and Škerget [14] (who employed a boundary element method for power-law fluids) and Barnoon and Toghraie [15] (who used a finite volume method to simulate pseudo-plastic non-Newtonian nanofluid $\left(\mathrm{Al}_{2} \mathrm{O}_{3}+\mathrm{CMC}\right)$ within a porous circular concentric region). Several excellent benchmark studies exist for numerical simulations of natural convection in enclosures including the work of de Vahl Davis [16] who simulated natural convection heat transfer in an air filled square cavity. A similar analysis has been conducted by Valencia and Frederick [17] with partially active walls. The role of partially heated verticals on heat transfer in air of square enclosure has been reported by Kane et al. [18]. Recent studies of natural or mixed convection in a square cavity filled with different fluids and with different supplementary effects (e.g. magnetic body forces, lid-driven boundary etc) have been presented in [19]-[25].

From a close inspection of the scientific literature, it is evident that time-dependent natural convection heat transfer in a square enclosure filled with Casson fluid over a wide range of Rayleigh numbers has not been studied so far either numerically or experimentally. In view of this, the present computational analysis aim is to study the convective heat transfer of nonNewtonian Casson fluid in a square enclosure under the transverse temperature gradients with a Marker and Cell (MAC) algorithm which features a finite difference scheme along with a staggered grid system. The projection method is employed to evaluate the pressure term. Extensive visualizations of the impact of emerging physical parameters (Rayleigh number, and Casson viscoplastic parameter) on streamline and isotherm distributions in the cavity are presented and Nusselt numbers also computed.

\section{MATHEMATICAL MODEL}

The physical regime under investigation comprises a two-dimensional square geometry. The square geometry enclosed by four thermal walls. The upper and lower horizontal walls are thermally insulated and the vertical walls are isothermal and prescribed different temperatures. The geometry analyzed is depicted in Fig. 1 and the working fluid in the enclosure is considered to be non-Newtonian fluid (i.e. Casson viscoplastic fluid). The vertical walls are orientated in the y-direction with unit length and the bottom and top walls are directed along the $\mathrm{x}$-direction with unit length. The enclosure vertical left and right walls are sustained at temperatures, $T_{h}$ and $T_{c}$ (i.e $T_{c}<T_{h}$ ) respectively. Except for the density, all properties of the Casson fluid are considered to be constant. In addition, the thermal buoyancy force is included through the Boussinesq approximation. The constitutive equation for a Casson fluid i.e. the stress-strain relation takes the form: 


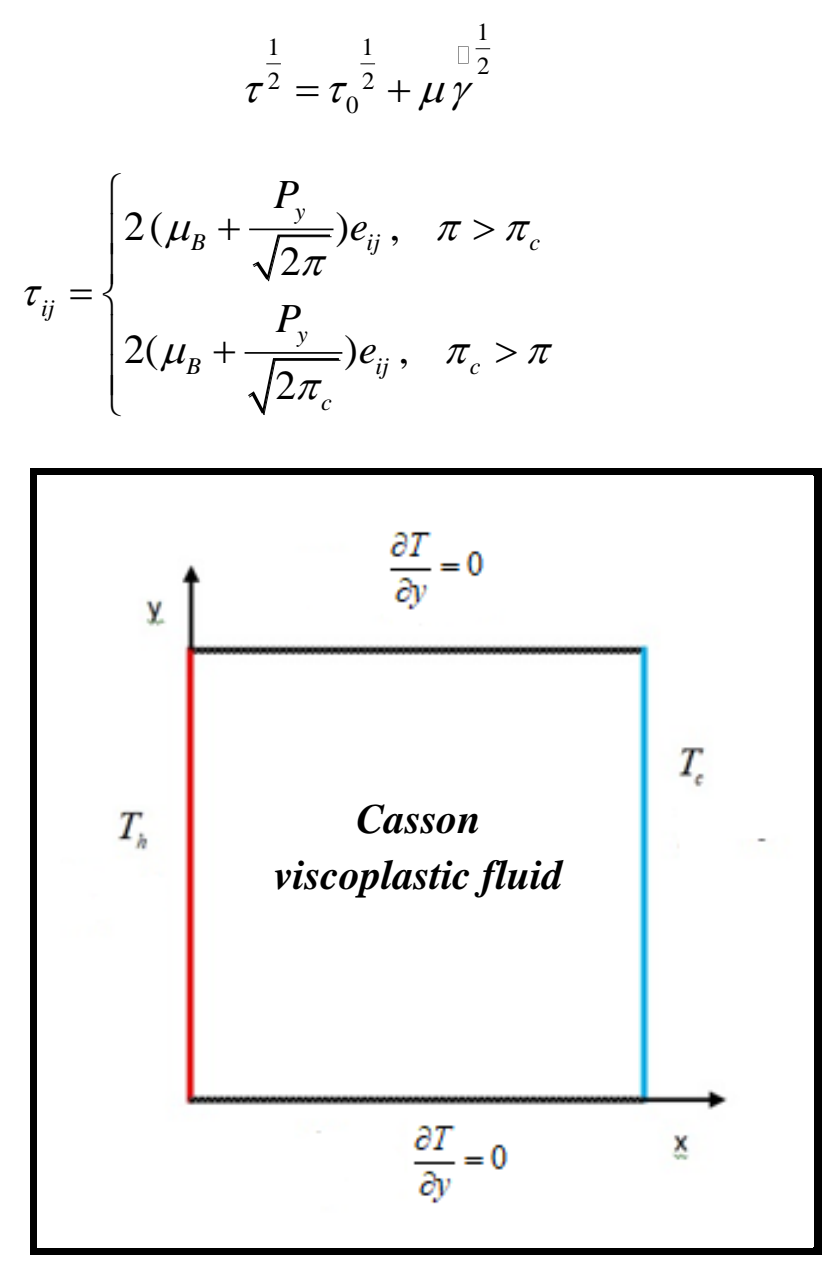

Fig. 1. Schematic of problem

Under the above assumptions, neglecting viscous heating, the governing equations for mass, momentum and energy for two-dimensional, unsteady, laminar, incompressible Casson fluid in a square cavity may be presented in vector form (with the appropriate term incorporated from Eqn. (1) for Casson rheological effect) as follows, with associated boundary conditions at the four walls of the enclosure:

$$
\begin{aligned}
& \nabla \cdot \boldsymbol{V}=0 \\
& \rho_{f}\left[\frac{\partial \boldsymbol{V}}{\partial t}+(\boldsymbol{V} \cdot \nabla) \boldsymbol{V}\right]=-\nabla p+\mu\left(1+\frac{1}{\gamma}\right) \nabla^{2} \boldsymbol{V}+\left[\rho_{f} \beta\left(T-T_{c}\right)\right] \boldsymbol{g}_{y} \\
& \frac{\partial T}{\partial t}+(\boldsymbol{V} \cdot \nabla) T=\alpha \nabla^{2} T \\
& t=0, u=v=T=0 \text { for } 0 \leq x \leq 1,0 \leq y \leq 1 \\
& t>0 \quad u=v=0, \frac{\partial T}{\partial y}=0 \text { at } \mathrm{y}=0,1
\end{aligned}
$$


$u=v=0, T=T_{h}$, at $\mathrm{x}=0$

$u=v=0, T=T_{c}$, at $\mathrm{x}=1$

It is judicious to introduce the following non-dimensional variables:

$$
\tau=\frac{t \alpha}{L^{2}},(X, Y)=\frac{(x, y)}{L}, U=\frac{u L}{\alpha}, V=\frac{v L}{\alpha}, P=\frac{p L^{2}}{\rho \alpha^{2}}, \theta=\frac{T-T_{c}}{T_{h}-T_{c}}
$$

Implementing Eqn. (6) in the primitive eqns. (2)-(5), the resulting non-dimensional governing equations of natural convective flow in the enclosure assume the form:

$$
\begin{aligned}
& \frac{\partial U}{\partial X}+\frac{\partial V}{\partial Y}=0 \\
& \frac{\partial U}{\partial \tau}+U \frac{\partial U}{\partial X}+V \frac{\partial U}{\partial Y}=-\frac{\partial P}{\partial X}+\left(1+\frac{1}{\gamma}\right) \operatorname{Pr}\left(\frac{\partial^{2} U}{\partial X^{2}}+\frac{\partial^{2} U}{\partial Y^{2}}\right) \\
& \frac{\partial V}{\partial \tau}+U \frac{\partial V}{\partial X}+V \frac{\partial V}{\partial Y}=-\frac{\partial P}{\partial Y}+\left(1+\frac{1}{\gamma}\right) \operatorname{Pr}\left(\frac{\partial^{2} V}{\partial X^{2}}+\frac{\partial^{2} V}{\partial Y^{2}}\right)+\operatorname{Ra} \cdot \operatorname{Pr} \theta \\
& \frac{\partial \theta}{\partial \tau}+U \frac{\partial \theta}{\partial X}+V \frac{\partial \theta}{\partial Y}=\frac{\partial^{2} \theta}{\partial X^{2}}+\frac{\partial^{2} \theta}{\partial Y^{2}}
\end{aligned}
$$

The non-dimensional wall boundary conditions emerge as:

$\tau=0 \quad U=V=\theta=0$ for $0 \leq X \leq 1, \quad 0 \leq Y \leq 1$

$\tau>0 \quad U=V=0, \frac{\partial \theta}{\partial Y}=0$ at $\mathrm{Y}=0,1$

$U=V=0, \theta=1$ at $\mathrm{Y}=0$

$U=V=0, \theta=0$ at $\mathrm{Y}=1$

In Eqns. (7)-(10), the non-dimensional governing parameters are Prandtl number, $\operatorname{Pr}=\frac{v}{\alpha}$, Rayleigh number, $R a=\frac{g \beta\left(T-T_{c}\right) L^{3} P r}{v^{2}}$ and Casson parameter, $\gamma$. The local and average heat transfer rates are defined respectively as:

$$
\begin{aligned}
& N u=-\left(\frac{\partial \theta}{\partial X}\right)_{X=0}, \\
& \overline{N u}=\int_{0}^{L} N u d Y,
\end{aligned}
$$




\section{MAC NUMERICAL SOLUTION AND VALIDATION}

The non-dimensional partial differential equations (7)-(10) under boundary conditions (11) are solved by the Marker and Cell (MAC) computational method. For this method we consider a uniform grid, i.e. the step length of both $X$ and $Y$ directions are equal $(\mathrm{dX}=\mathrm{dY})$. The discretization of the convective terms and diffusion term is conducted via a central difference finite scheme. The Poisson equation of stream function is solved by an effective iterative method known as successive over-relaxation (SOR). The vector form of the momentum equations (8) and (9) is:

$\frac{\partial \overline{\boldsymbol{V}}}{\partial \tau}+(\overline{\boldsymbol{V}} \cdot \nabla) \overline{\boldsymbol{V}}=-\nabla p+\operatorname{Pr}(1+K) \nabla^{2} \overline{\boldsymbol{V}}+\operatorname{Ra} \operatorname{Pr} \theta \bullet g$

where $K=\frac{1}{\gamma}, \quad \bar{V}=[U V]$ and $g=\left[\begin{array}{l}0 \\ 1\end{array}\right]$. In compact form the momentum Eqn

becomes:

$\frac{\partial \overline{\boldsymbol{V}}}{\partial \tau}+A=-\nabla p+B+C$

Where

$$
\begin{aligned}
& A=(\overline{\boldsymbol{V}} \cdot \nabla) \overline{\boldsymbol{V}} \\
& B=\operatorname{Pr}(1+K) \nabla^{2} \overline{\boldsymbol{V}} \\
& C=\operatorname{Ra} \operatorname{Pr} \theta \bullet g
\end{aligned}
$$

Next the discrete vector equation across a step takes the form:

$$
\frac{{\overline{\boldsymbol{V}_{i, j}}}^{k+1}-\overline{\boldsymbol{V}}_{i, j}^{k}}{d t}=-A_{i, j}^{k}-\nabla p_{i, j}^{k}+B_{i, j}^{k}+C_{i, j}^{k}
$$

With the constraint on velocity as follows:

$$
\nabla \mathrm{g} \overline{\boldsymbol{V}}_{i, j}^{k+1}=0
$$

Splitting Eqn. (15) we have:

$$
\frac{{\overline{\boldsymbol{V}_{i, j}}}^{*}-\overline{\boldsymbol{V}}_{i, j}^{k}}{d t}=-A_{i, j}^{k}+B_{i, j}^{k}+C_{i, j}^{k}
$$

and 
$\frac{{\overline{\boldsymbol{V}_{i, j}}}^{k+1}-{\overline{\boldsymbol{V}_{i, j}}}^{*}}{d t}=-\nabla p_{i, j}^{k}$

${\overline{\boldsymbol{V}_{i, j}}}^{k+1}={\overline{\boldsymbol{V}_{i, j}}}^{*}-d t \cdot \nabla p_{i, j}^{k}$

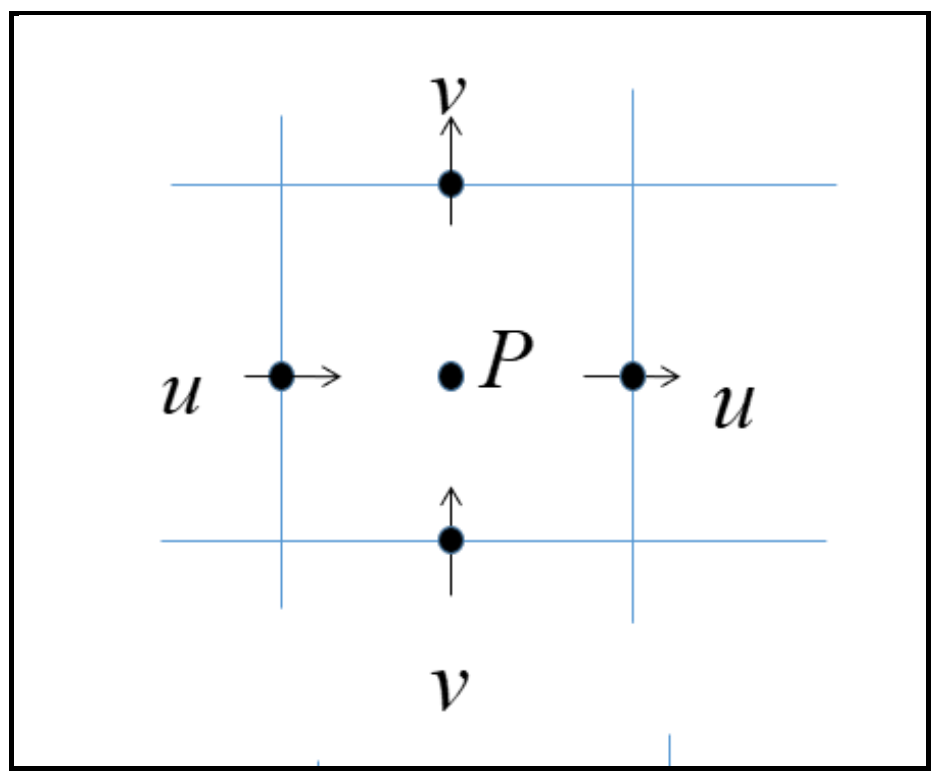

Fig. 2. Staggered MAC grid

Here the intermediate velocity is introduced by ${\overline{V_{i, j}}}^{*}$, To get the pressure Poisson equation, we apply the divergence of Eqn. (19) and using the mass conservation equation which yields:

$$
\begin{aligned}
& \nabla \cdot{\overline{\boldsymbol{V}_{i, j}}}^{k+1}=\nabla \cdot{\overline{\boldsymbol{V}_{i, j}}}^{*}-d t(\nabla \cdot \nabla) p_{i, j}^{k} \\
& 0=\nabla \cdot \overline{\boldsymbol{V}}_{i, j}^{*}-d t(\nabla \cdot \nabla) p_{i, j}^{k} \\
& \frac{1}{d t} \nabla \cdot{\overline{\boldsymbol{V}_{i, j}}}^{*}=\nabla^{2} p_{i, j}^{k}
\end{aligned}
$$

Equation (22) can be solved by the iterative method of successive over relaxation (SOR) and the final velocities are obtained as follows:

${\overline{\boldsymbol{V}_{i, j}}}^{k+1}={\overline{\boldsymbol{V}_{i, j}}}^{*}-d t \cdot \nabla p_{i, j}$

Defining a dimensional stream function by the Cauchy-Riemann equations:

$$
U=\frac{\partial \psi}{\partial Y} \text { and } V=-\frac{\partial \psi}{\partial X}
$$

The temperature equation is solved as follows: 


$$
\begin{gathered}
\frac{\theta^{n+1}{ }_{i+1, j}-\theta_{i-1, j}^{n}}{d t}=-\frac{\partial \psi}{\partial Y} \frac{\partial \theta}{\partial X}+\frac{\partial \psi}{\partial X} \frac{\partial \theta}{\partial Y}+\nabla^{2} \theta \\
\theta^{n+1}{ }_{i+1, j}=\theta_{i-1, j}^{n}+d t\left\{-\frac{\partial \psi}{\partial Y} \frac{\partial \theta}{\partial X}+\frac{\partial \psi}{\partial X} \frac{\partial \theta}{\partial Y}+\nabla^{2} \theta\right\}^{n}
\end{gathered}
$$

The MAC method has been coded in MATLAB symbolic software and validated with earlier studies for $\operatorname{Pr}=0.71$ (air) and the Newtonian case $(\gamma \rightarrow 0)$ for a range of Rayleigh numbers. Table. 1 and Table. 2 provide good corroboration of the MAC solutions for local Nusselt number with these benchmark studies by de Vahl Davis [16] and Wan et al. [25]. High confidence in the MAC code is therefore achieved.

Table. 1. Comparison of local Nusselt number.

\begin{tabular}{|c|c|c|}
\hline \multirow{2}{*}{} & \multicolumn{2}{|c|}{$N u$} \\
\cline { 2 - 3 } & MAC Results & de Vahl Davis [16] \\
\hline $\mathrm{Ra}=10^{4}$ & 2.2526 & 2.242 \\
$\mathrm{Ra}=10^{5}$ & 4.5907 & 4.523 \\
$\mathrm{Ra}=10^{6}$ & 8.9905 & 9.035 \\
\hline
\end{tabular}

Table. 2. Comparison of local Nusselt number.

\begin{tabular}{|c|c|c|}
\hline \multirow{2}{*}{} & \multicolumn{2}{|c|}{$N u$} \\
\cline { 2 - 3 } & MAC Results & Wan et al. [25] \\
\hline $\mathrm{Ra}=10^{4}$ & 2.2526 & 2.254 \\
$\mathrm{Ra}=10^{5}$ & 4.5907 & 4.598 \\
$\mathrm{Ra}=10^{6}$ & 8.9905 & 8.976 \\
\hline
\end{tabular}

\section{RESULTS AND DISCUSSION}

All MAC MATLAB code simulations have been executed for buoyancy driven flow of nonNewtonian Casson fluid with heating of side wall. A Prandtl number $\operatorname{Pr}=6.8$ is considered throughout analysis which is appropriate for non-Newtonian polymeric fluids as noted by Incropera and De Witt [26]. Isotherms and streamline distributions (flow patterns) inside the square cavity have been plotted in Figs. 3-13. Herein, a comprehensive analysis of Casson fluid parameter and thermal Rayleigh number effects on the contours of flow patterns and temperature is conducted. The local and average Nusselt numbers are also computed. 
Fig.3. illustrates the effect of Casson fluid parameter $(\gamma)$ on flow patterns and temperature distribution inside the square cavity for Rayleigh number $\mathrm{Ra}=10^{3}$. For all the values of Casson fluid parameter considered, a single enlarged circular eddy is developed within the enclosure which is induced due to flow rising along the hot wall and descending along the cold wall with low thermal Rayleigh number. The corresponding isotherms are also modified with a variation in Casson fluid parameter. At $\gamma=0.1$, the isotherms are parallel to gravity as well as normal to the horizontal walls. With greater Casson fluid parameter up to1 the isotherms are morphed from the parallel topologies while the isotherms slowly migrate from the hot zone to the colder zones of the enclosure.

The influence of Casson fluid parameter on streamlines and temperature distributions for $R a=10^{4}$ is presented in Fig.4. The flow circulation changes and demonstrates the sensitivity of the flow structure to Casson fluid parameter i.e. rheology of the working fluid. The single enlarged circular eddy is adjusted from a dominant circular motion to a diagonal motion when increasing the Casson fluid parameter and this is associated with the domination of thermal convection by thermal conduction in the regime. The flow in the enclosure is accelerated with greater Rayleigh number whereas the flow is decelerated i.e. fluid velocity is damped by reducing (from 1 to 0.1 ) the Casson fluid parameter. Regardless of Rayleigh number, the fluid particle velocity is strongly regulated by the Casson fluid parameter. The corresponding isotherms exhibit gradual changes with increasing Casson fluid parameter from 0.1 to 1 . The isotherms occupy a uniformly whole area of the enclosure for low values of $\gamma$. With elevation in Casson fluid parameter the density of the temperature contours is increased in the vicinity of the top portion of the right wall and also the bottom portion of left wall. Heat transfer rate is enhanced at high values of the Casson fluid parameter and heat transfer rate is reduced at low value of Casson fluid parameter.

Fig. 5 illustrates the influence of Casson fluid parameter, $\gamma$ on streamline patterns and temperature distributions within the square enclosure for Rayleigh number, $\mathrm{Ra}=10^{5}$. Streamlines circulation within the enclosure is markedly changed when varying the Casson fluid parameter. In addition, the heat transfer rate is also controlled by the Casson fluid parameter. The streamlines demonstrate a circular structure for low $\gamma$ and the circulation is shifted laterally when increasing $\gamma$. Accordingly, the isotherms also are altered. Generally, thermal convection is more intense at high values of Rayleigh number. For almost all the cases the isotherms are stretched towards the cold wall and form a thermal boundary layer at the bottom of the hot wall and top of the left wall. The strong thermal boundary layer is developed when the fluid circulation is stretched horizontally. Heat transfer is also modified substantially and is higher from the hot wall to the cold wall.

The effect of Casson fluid parameter on streamline and temperature distributions at a higher value of Rayleigh number $R a=10^{6}$ are depicted in Fig. 6. For higher values of $R a$, the velocity of fluid and convective heat transfer intensity are increased. Fluid circulation exhibits a horizontal elliptical eddy shape for all values of Casson fluid parameter. The density of streamlines are increased with greater Casson fluid parameter values up to 1 . The isotherms distribution occupies the entire area of the enclosure. A strong thermal plume is developed 
along the hot and cold walls while these isotherms are clustered at the opposite potions of the hot and cold wall. The steeper isotherms are diminished, and a more prominent thermal boundary layer is formed along the hot and cold wall with increasing Casson fluid parameter. As a result, the isotherms are parallel to the horizontal walls in the middle (core) of the enclosure.

Mid-section velocity profile variations with the Casson fluid parameter for various values of Rayleigh number are presented in the Fig.7 and Fig.8 Regardless of Rayleigh number the fluid velocities along $X$ and $Y$-directions are increased for greater values of Casson fluid parameter. These velocity profiles are symmetric at the line $Y=0.5$ and the line $X=0.5$ respectively.

Fig.9 and Fig.10 present the effects of Rayleigh number on non-dimensional velocity components, $(U, V)$ along the vertical mid-plane and horizontal mid-plane respectively, for Casson fluid parameter, $\gamma=0.1$. The fluid velocity profiles are gradually enhanced as thermal Rayleigh number rises i.e. significant flow acceleration in both $X, Y$ directions is induced.

Figs. 11-13 illustrate the Nusselt number (local and average) distributions for various parameters at the hot wall. Fig.11 illustrates the local Nusselt number variations along the hot wall (right vertical boundary) for different values of Casson fluid parameter and different values of Rayleigh number. These results show that heat transfer rate along the hot wall is modified with both Casson rheological parameter, $\gamma$ and Rayleigh number. Low heat transfer rate is observed at $R a=10^{3}$ and higher heat transfer rate is noticed at $R a=10^{6}$. However, the heat transfer rate is increased with rising of $\gamma$ for any value of Rayleigh number. In addition, the local Nusselt number along the hot surface is enhanced with increasing $R a$ values at $\gamma=0.1$ implying that stronger thermal buoyancy encourages heat transfer rates. It is also apparent in Fig.12. that the average Nusselt number along the hot wall is significantly boosted with increasing Rayleigh number. Fig.13 illustrates that the average Nusselt number is elevated with both increasing Rayleigh number and Casson fluid parameter, although the latter effect is decidedly more prominent at higher Rayleigh numbers.

\section{CONCLUSIONS}

The transport phenomena in natural convection of a Casson rheological fluid within a square enclosure with isothermal side walls and insulated horizontal walls. has been studied numerically with a MAC algorithm coded in MATLAB. A finite difference scheme and staggered grid have been used for the computational solution of the non-dimensional partial differential conservation equations with associated boundary conditions. Validation of the code with earlier Newtonian simulations has been included. Extensive graphical plots have been presented for streamline, temperature and Nusselt number (local and average) distributions. The computations have shown that:

1. Increasing thermal Rayleigh number $R a$ leads to an increase in thermal convection intensity and heat transfer regardless of the value of the Casson fluid parameter. 
2. For all Rayleigh numbers, the flow and heat transfer rate is enhanced with increasing Casson fluid parameter.

3. The temperature contours are more clustered along the hot and cold walls for higher value of Rayleigh number.

4. Increasing Rayleigh number enhances the local Nusselt number.

5. Increasing both Rayleigh number and Casson fluid parameter results in an elevation in average Nusselt number.

The present simulations have examined a simple viscoplastic model with the efficient MAC numerical approach. Future enclosure simulation investigations may consider alternate nonNewtonian models e.g. micropolar liquids [27] and efforts in this regard are currently underway.

\section{REFERENCES}

[1] Shaw, S., Gorla, R.S.R., Murthy, P.V.S.N. and Ng, C.O., Pulsatile Casson fluid flow through a stenosed bifurcated artery, International Journal of Fluid Mechanics Research, 36 (2009) 43-63.

[2] Zaib, A., Bhattacharyya, K., Uddin, M.S. and Shafie, S, Dual solutions of non-Newtonian Casson fluid flow and heat transfer over an exponentially permeable shrinking sheet with viscous dissipation, Modeling and Simulation in Engineering, 2016 (2016) 1-8.

[3] V.R. Prasad, A. SubbaRao, N. Bhaskar Reddy, B. Vasu, O. Anwar Bég, Modelling laminar transport phenomena in a Casson rheological fluid from a horizontal circular cylinder with partial slip, Proc IMechE- Part E: J. Process Mechanical Engineering, 227 (2013) 309-326.

[4] M. Das., R. Mahato., R. Nandkeolyar., Newtonian heating effect on unsteady hydromagnetic Casson fluid flow past a flat plate with heat and mass transfer. Alexandria Engineering Journal, 54(4) (2015) 871-879.

[5] G. J. Reddy, B. Kethireddy, M. Kumar and O. Anwar Bég, Transient analysis of Casson fluid thermo-convection from a vertical cylinder embedded in a porous medium: entropy generation and thermal energy transfer visualization, Journal of Central South University Science \& Technology of Mining and Metallurgy, 26 (5) 1342-1361 (2019).

[6] S. Ganganapalli, S. Kata and V. Bhumarapu., Unsteady boundary layer flow of a Casson fluid past a wedge with wall slip velocity, Journal of Heat and Mass Transfer Research, 4 (2017) 91-102.

[7] B. Vasu, Atul Kumar Ray, O. Anwar Bég and Rama Subba Reddy Gorla, Magnetobioconvection flow of a Casson thin film with nanoparticles over an unsteady stretching sheet: HAM and GDQ computation, Int. J. Numerical Methods Heat Fluid Flow (2019). 33 pages. doi.org/10.1108/HFF-02-2019-0158

[8] N. S. Akbar, D. Tripathi, O. Anwar Bég, Z. H. Khan, MHD dissipative flow and heat transfer of Casson fluids due to metachronal wave propulsion of beating cilia with thermal and velocity slip effects under an oblique magnetic field, Acta Astronautica, 128 (2016) 1-12. 
[9] S. Kuharat, O. Anwar Bég, Ali Kadir and M. Babaie, Computational fluid dynamic simulation of a solar enclosure with radiative flux and different metallic nano-particles, International Conference on Innovative Applied Energy (IAPE'19), Oxford, United Kingdom, 14-15 March (2019).

[10] W.E. Langlois, Buoyancy-driven flows in crystal-growth melts, Annual Review of Fluid Mechanics, 17 (1985) 191-215.

[11] Z. Alloui, P. Vasseur., Natural convection of Carreau-Yasuda non-Newtonian fluids in a vertical cavity heated from the sides, International Journal of Heat and Mass Transfer, 84 (2015) 912-924.

[12] GH. R. Kefayati, Simulation of non-Newtonian molten polymer on natural convection in a sinusoidal heated cavity using FDLBM, Journal of Molecular Liquids, 195 (2014) 165-174.

[13] GH. R. Kefayati., FDLBM simulation of magnetic field effect on mixed convection in a two-sided lid-driven cavity filled with non-Newtonian nanofluid, Powder Technology, 280 (2015) 135-153.

[14] R. Jecl, L. Škerget, Boundary element method for natural convection in non-Newtonian fluid saturated square porous cavity, Engineering Analysis with Boundary Elements, 27 (2003) 963-975.

[15] Pouya Barnoon, Davood Toghraie., Numerical investigation of laminar flow and heat transfer of non-Newtonian nanofluid within a porous medium, Powder Technology, 325 (2018) 78-91.

[16] De Vahl Davis, G., Natural convection of air in a square cavity: a benchmark numerical solution. International Journal for Numerical Methods in Fluids, 3 (1983) 249-264.

[17] Valencia, A. and Frederick, R.L., Heat transfer in square cavities with partially active vertical walls. International Journal of Heat and Mass Transfer, 32 (1989) 1567-1574.

[18] M. K. Kane, Cheikh Mbow, Mamadou Lamine Sow, Joseph Sarr., A study on natural convection of air in a square cavity with partially thermally active side walls, Open Journal of Fluid Dynamics, 7 (2017) 623-641.

[19] P. Kandaswamy, M. Muthtamilselvan, J Lee, Prandtl number effects on mixed convection in a lid-driven porous cavity. J Porous Media 11 (2008) 791-801.

[20] K.Venkatadri, S. Gouse Mohiddin and M. Suryanarayana Reddy, Numerical analysis of unsteady MHD mixed convection flow in a lid-driven square cavity with central heating on left vertical wall, Applications of Fluid Dynamics, Lecture Notes in Mechanical Engineering, Chapter 26 (2018) 355-370.

[21] B MD Hidayathulla Khan, K Venkatadri, O. Anwar Bég, V.Ramachandra Prasad, B. Mallikarjuna. Natural convection in a square cavity with uniformly heated and/or insulated 
walls using Marker-and-Cell Method, International Journal of Applied and Computational Mathematics, 4 (2018) 61.

[22] Venkatadri K, GouseMohiddin S, Suryanarayana Reddy M., Hydromagnetic quadratic natural convection on a lid-driven square cavity with isothermal and non-isothermal bottom wall, Engineering Computations, 34 (8) (2017) 2463-2478.

[23] K.Venkatadri, S. GouseMohiddin, M. Suryanarayana Reddy, Mathematical modeling of unsteady MHD double-diffusive natural convection flow in a square cavity, Frontiers in Heat and Mass Transfer, 9 (2017) No.33.

[24] T. Saitoh, K. Hirose, High - accuracy benchmark solutions to natural convection in a square cavity, Computational Mechanics, 4 (1989) 417-427.

[25] D. C. Wan, B. S. V. Patnaik, and G. W. Wei, A new benchmark quality solution for the buoyancy-driven cavity by discrete singular convolution, Numerical Heat Transfer, Part B, 40 (2001) 199- 228.

[26] F.P. Incropera and D. De Witt, Fundamentals of Heat and Mass Transfer, John Wiley and Sons, New York, $4^{\text {th }}$ edn (1996).

[27] R. Bhargava, Sharma, S., Bhargava, P., Anwar Bég, O., Kadir, A.: Finite element simulation of nonlinear convective heat and mass transfer in a micropolar fluid-filled enclosure with Rayleigh number effects. Int. J. Appl. Comput. Math.3 (2) 1347-1379 (2017). 
FIGURES

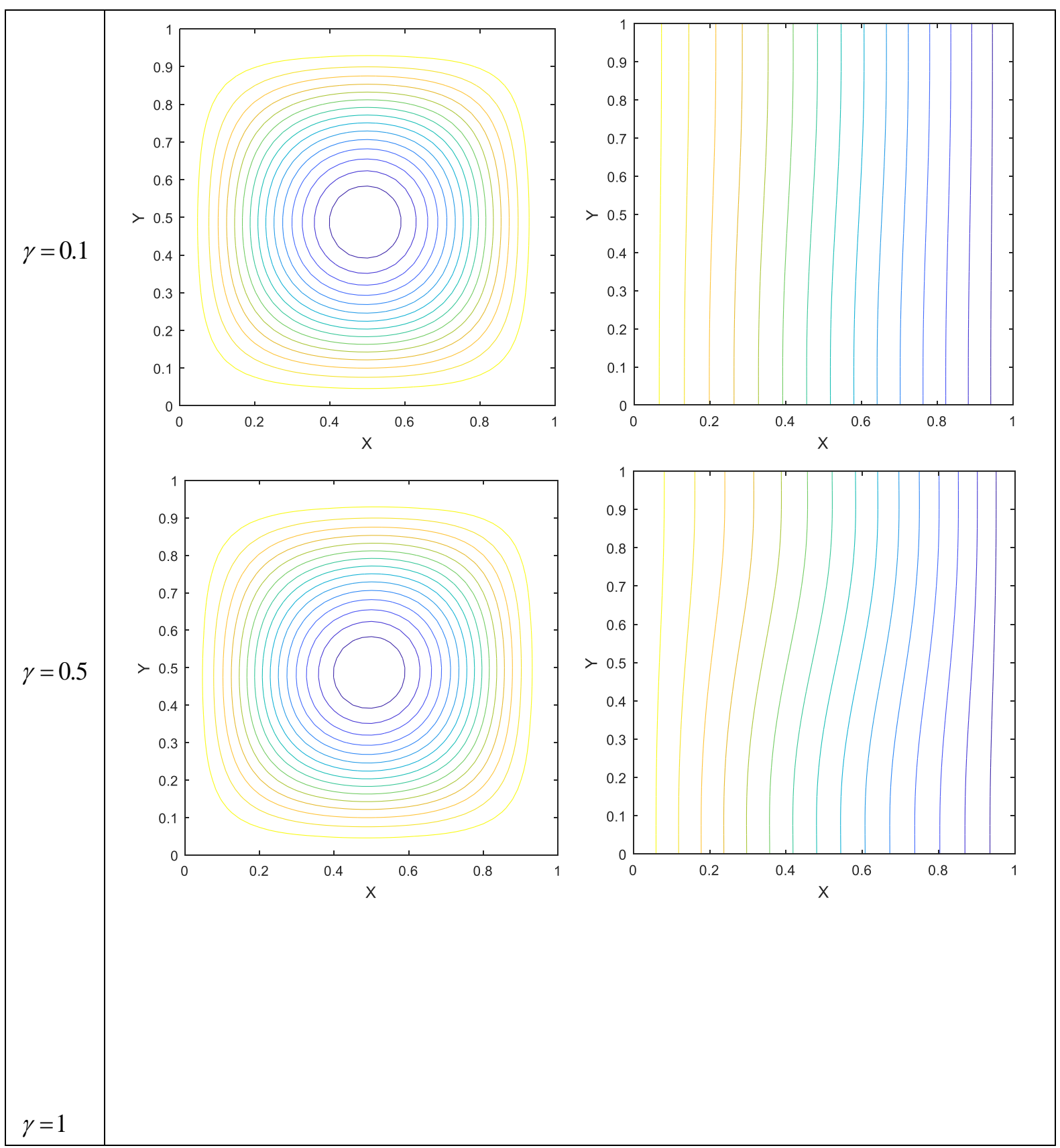




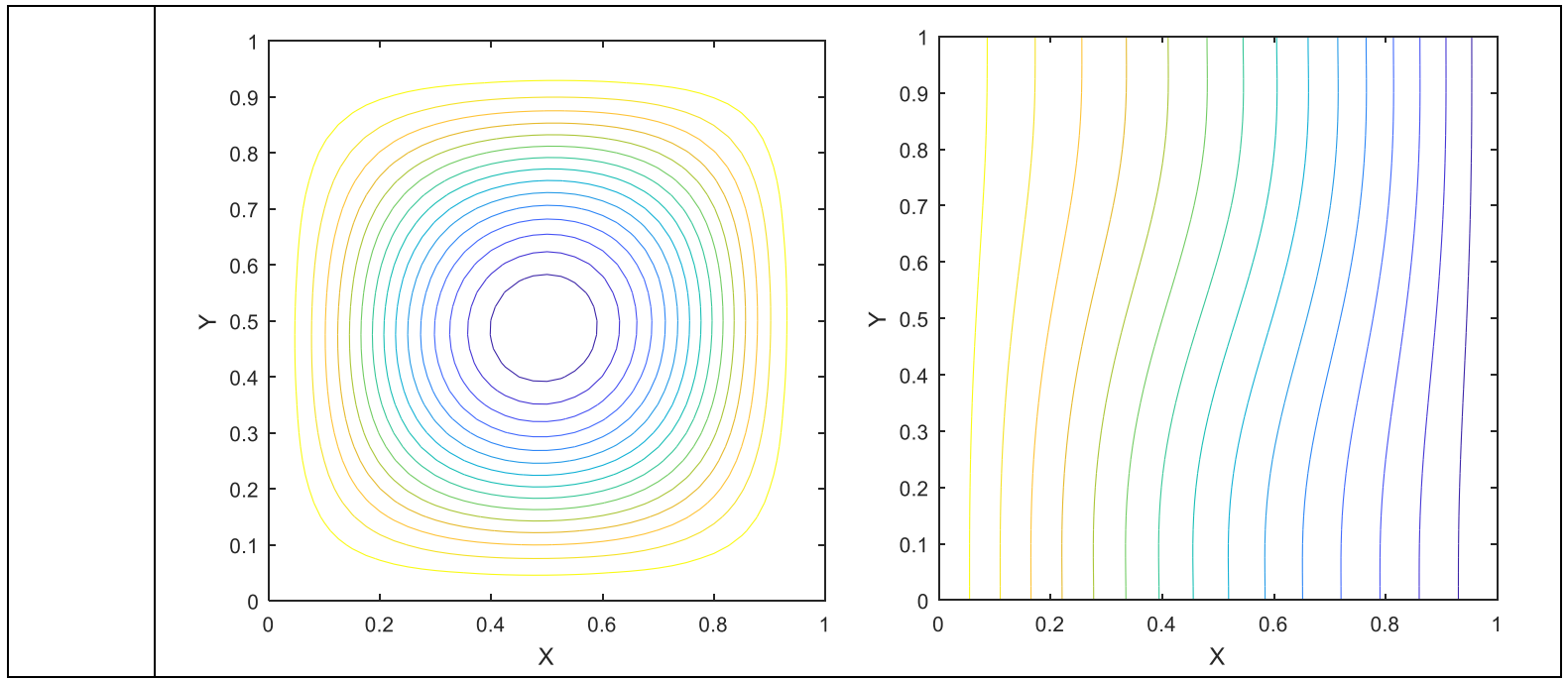

Fig. 3. Effect of Casson fluid parameter $(\gamma)$ on streamlines and isotherms for $R a=10^{3}$.

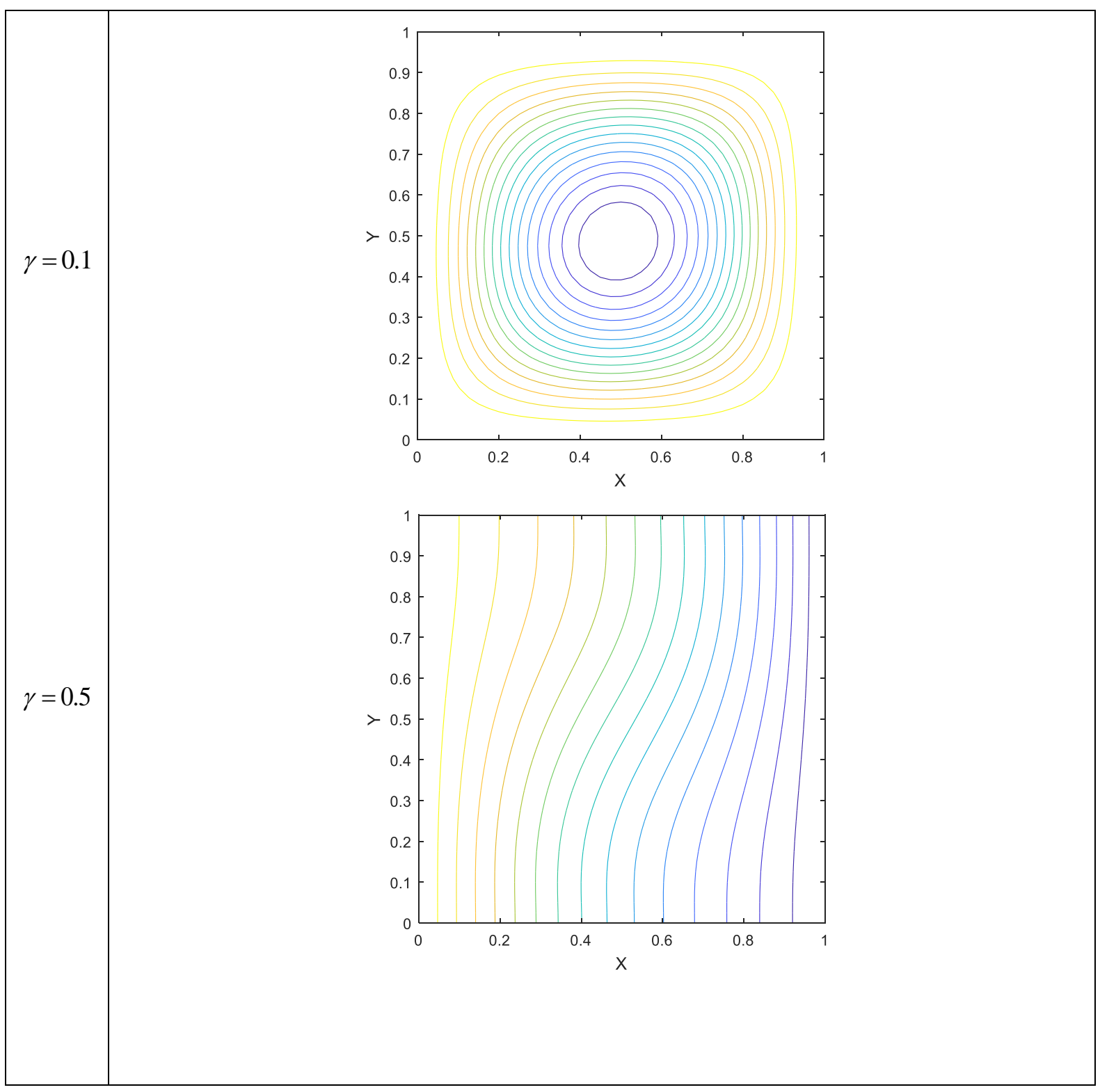




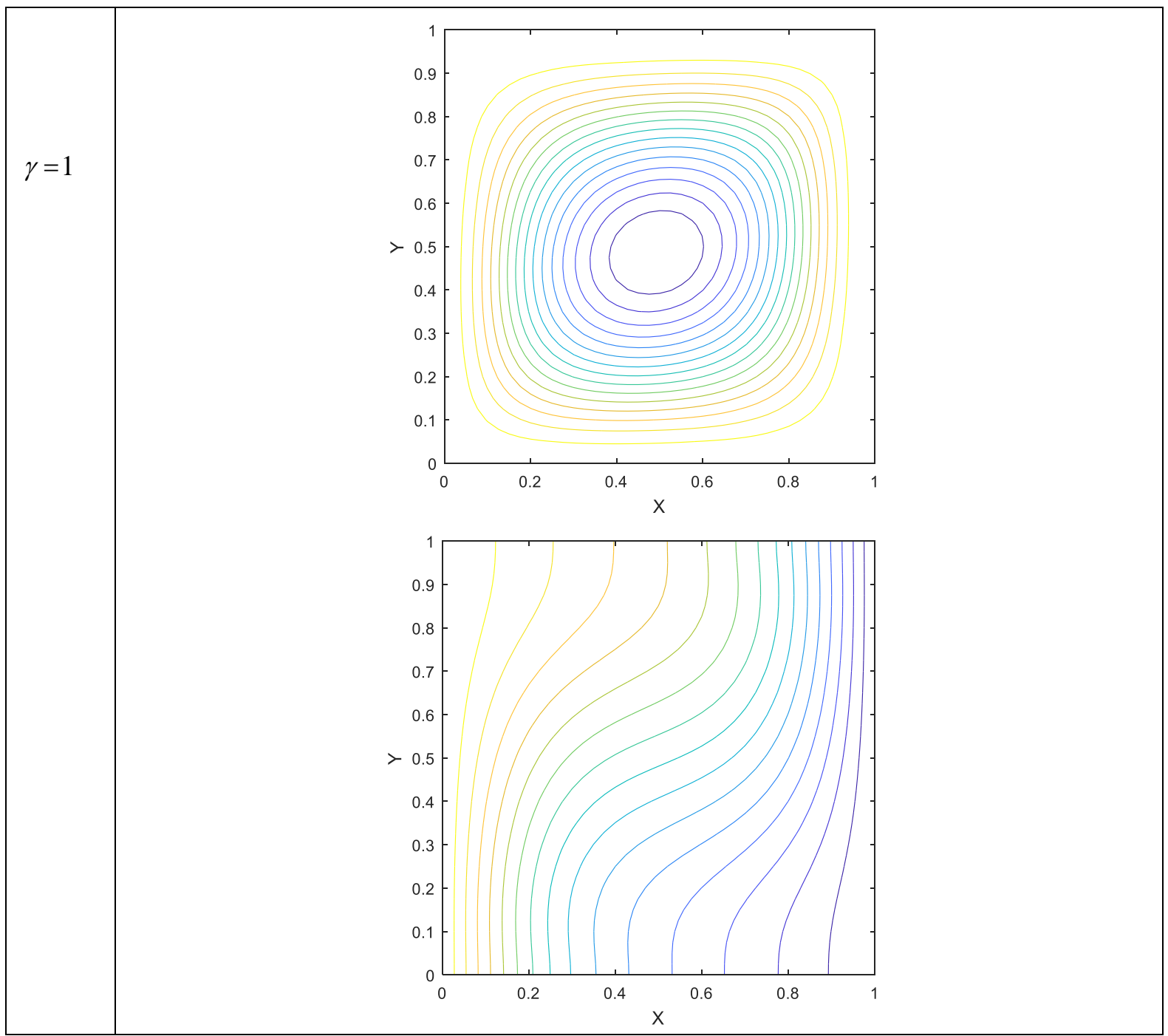




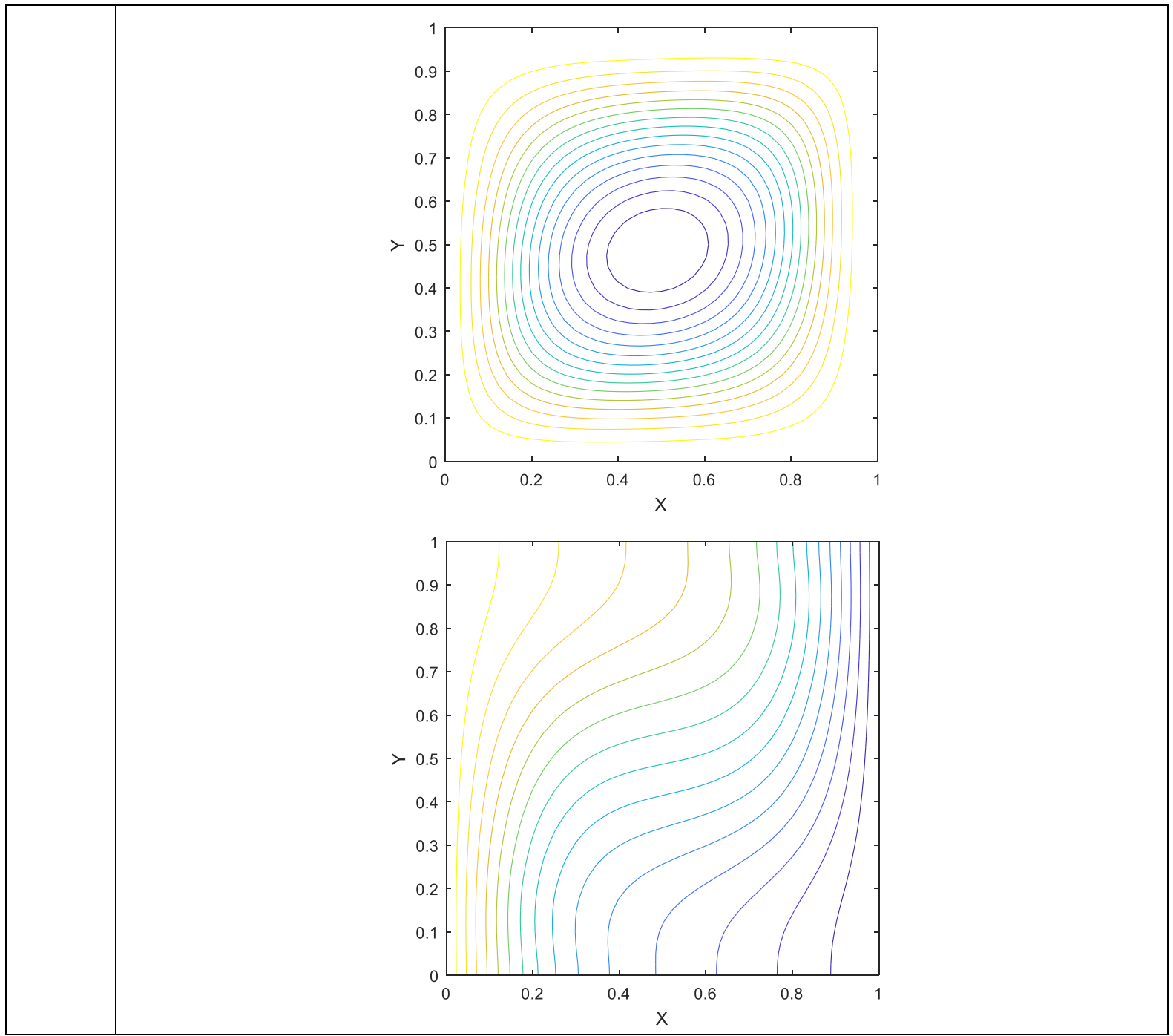

Fig. 4. Effect of Casson fluid parameter $(\gamma)$ on streamlines and isotherms for $R a=10^{4}$. 


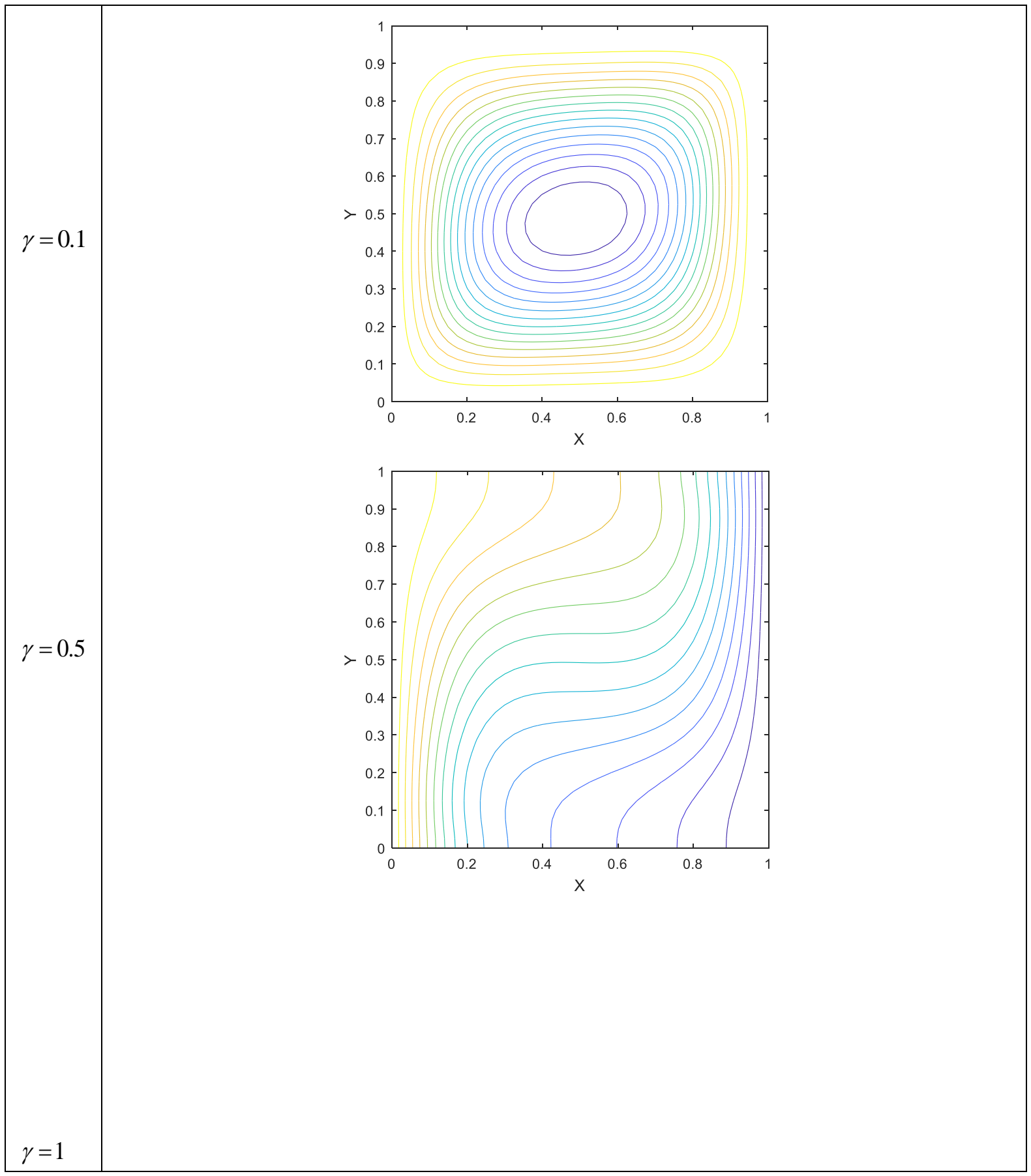




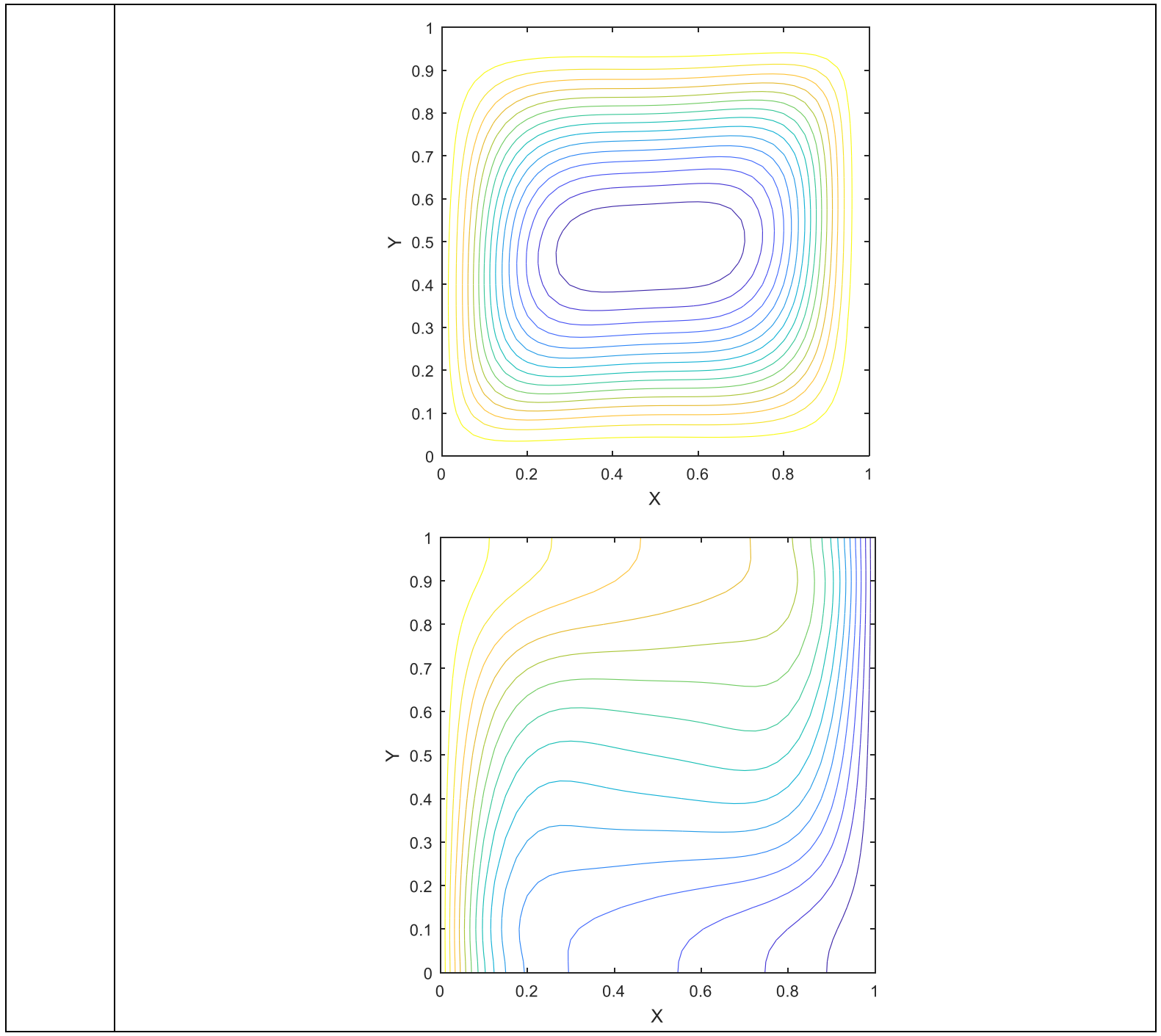




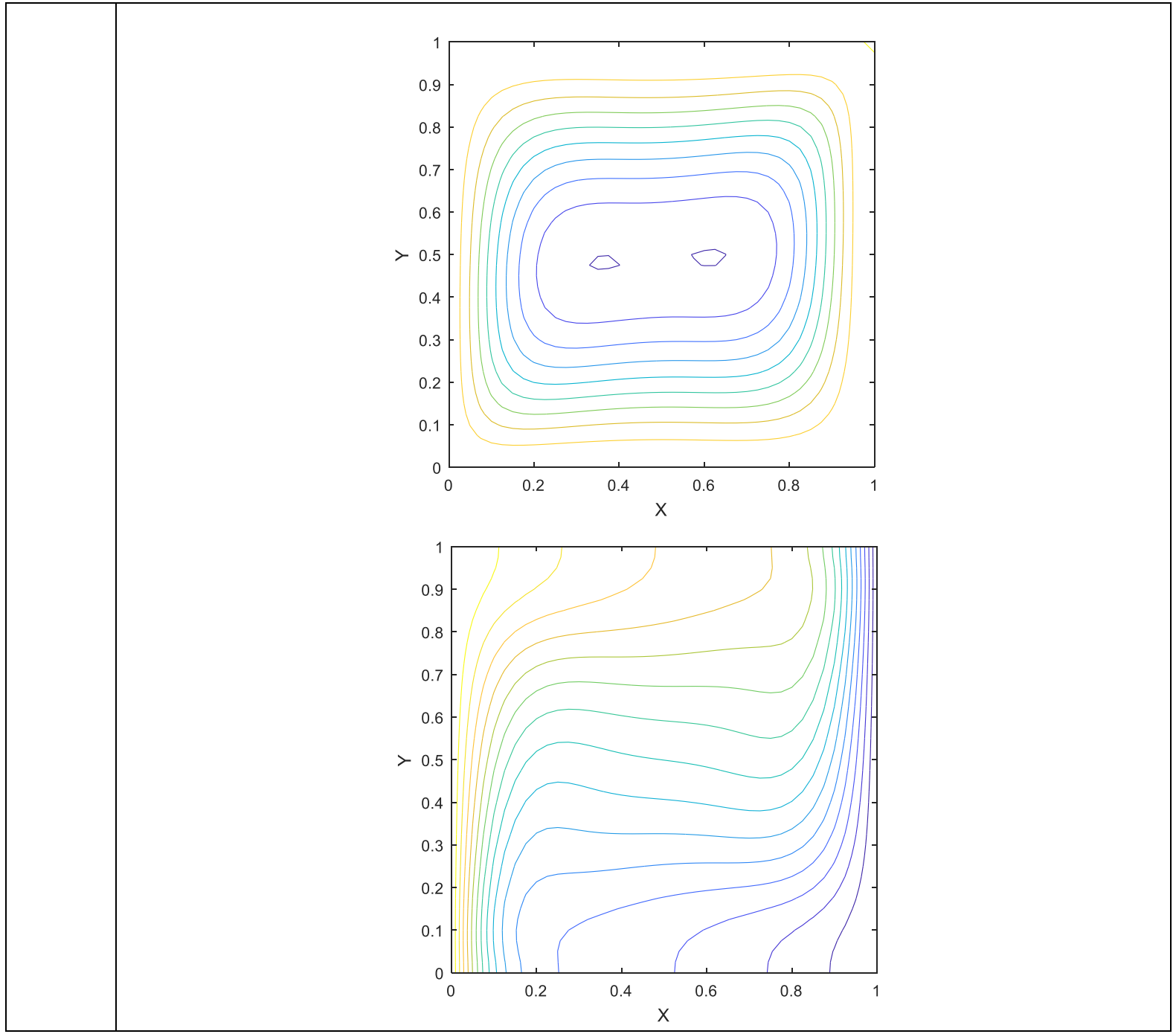

Fig. 5. Effect of Casson fluid parameter $(\gamma)$ on streamlines and isotherms for $R a=10^{5}$ 


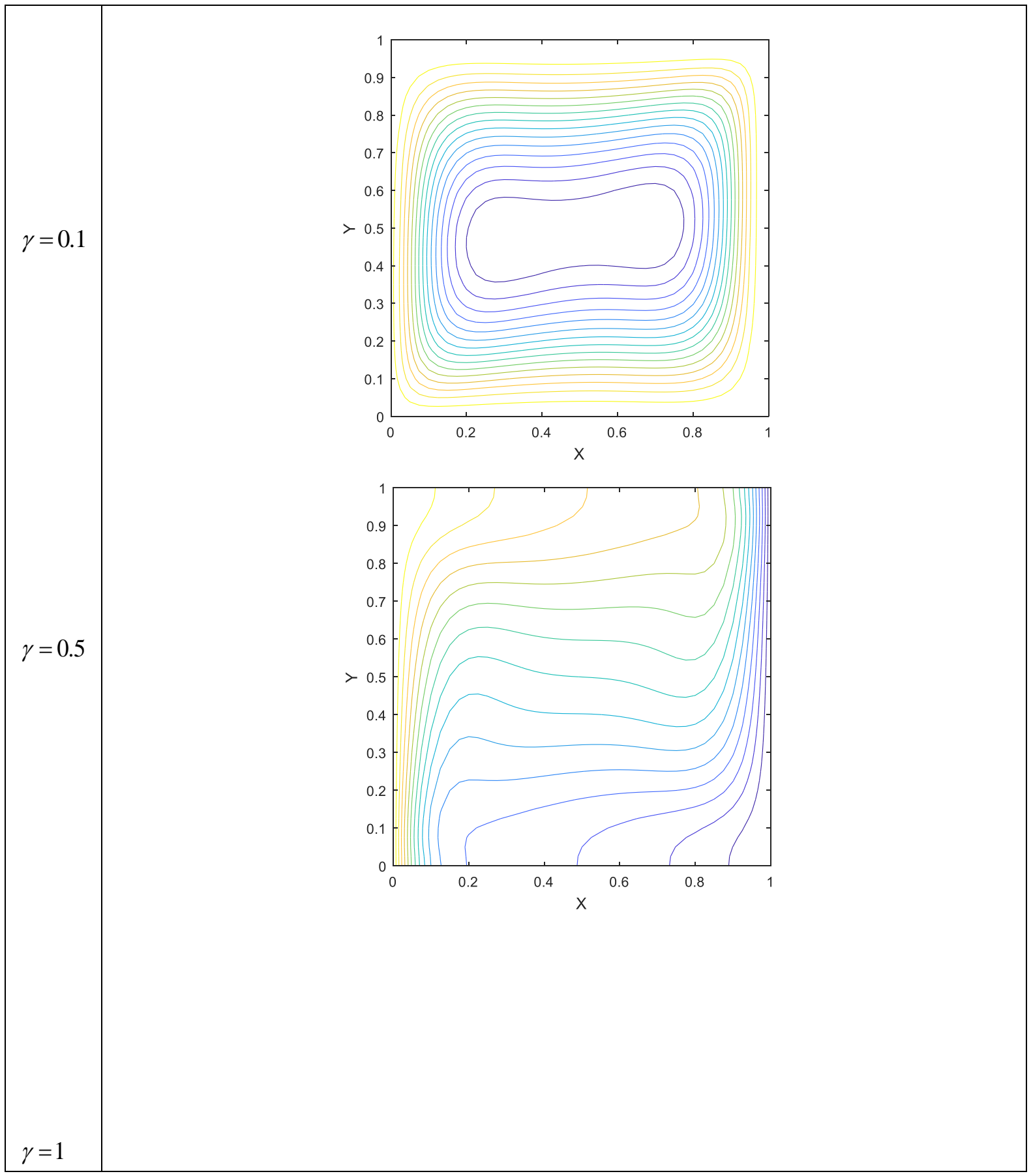




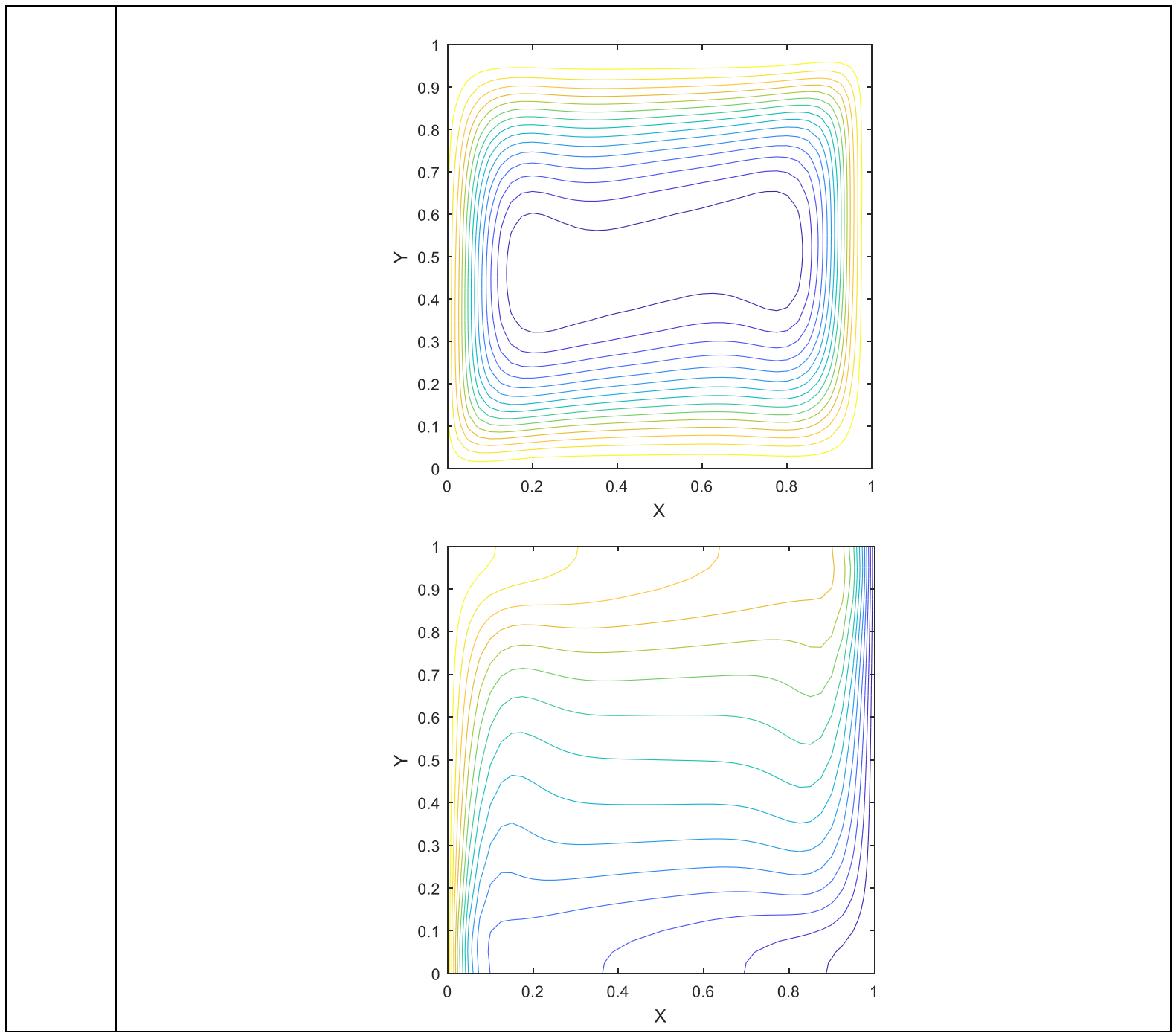




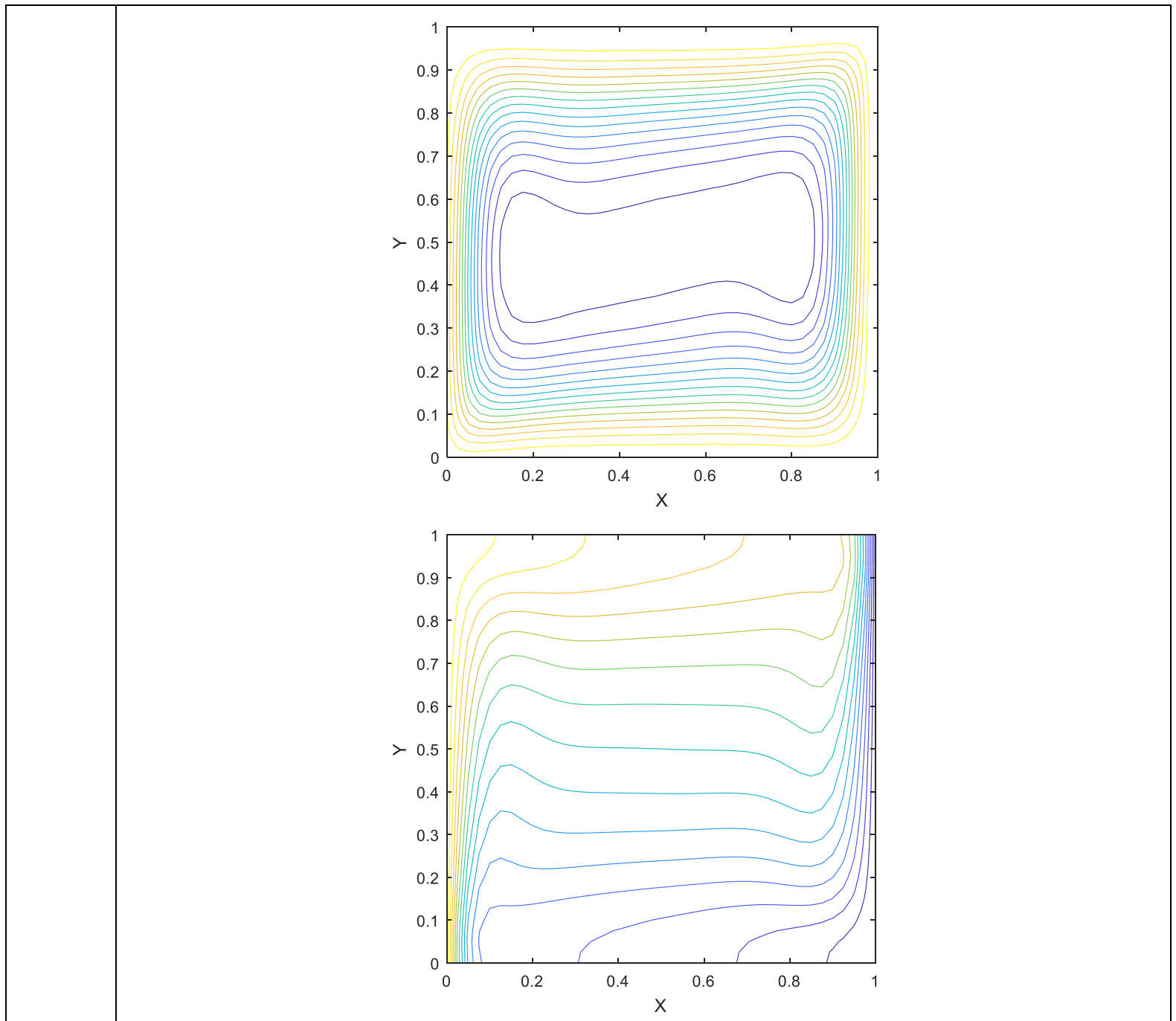

Fig. 6. Effect of Casson fluid parameter $(\gamma)$ on streamlines and isotherms for $R a=10^{6}$.

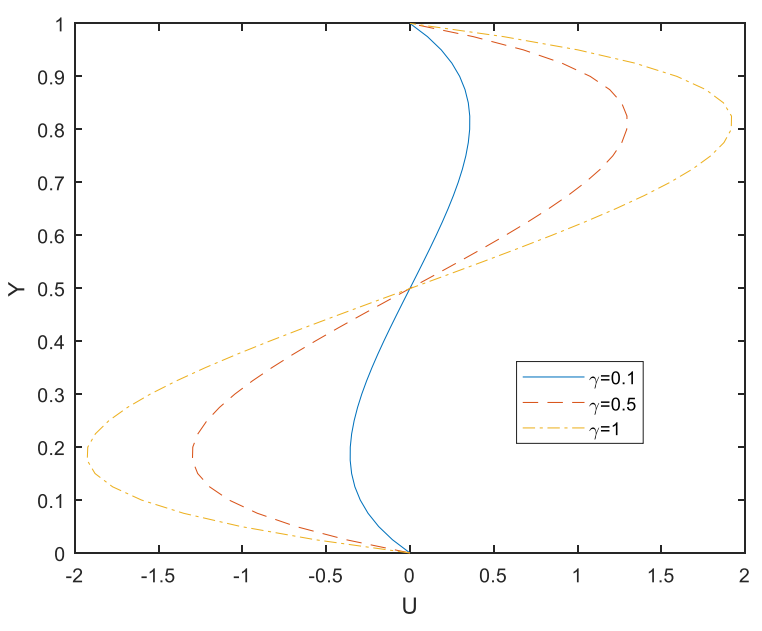

a. $\mathbf{R a}=10^{3}$

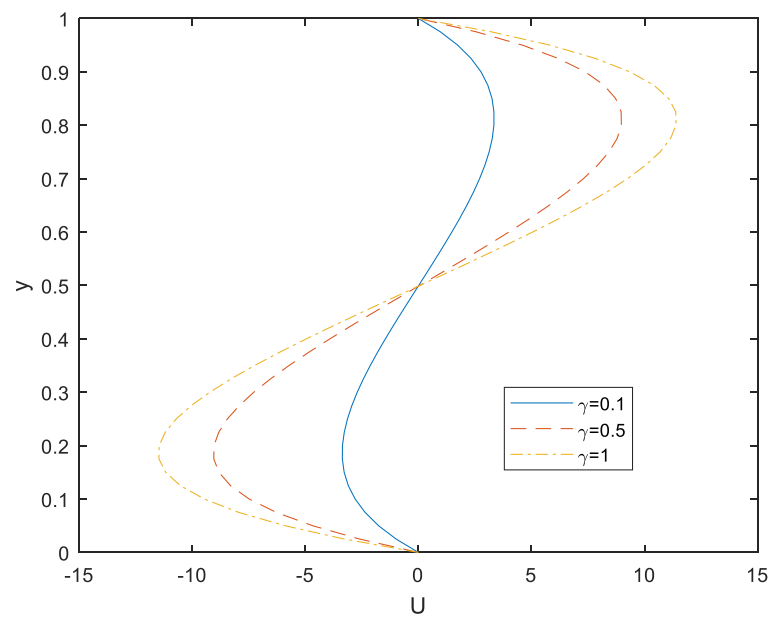

b. $R a=10^{4}$ 


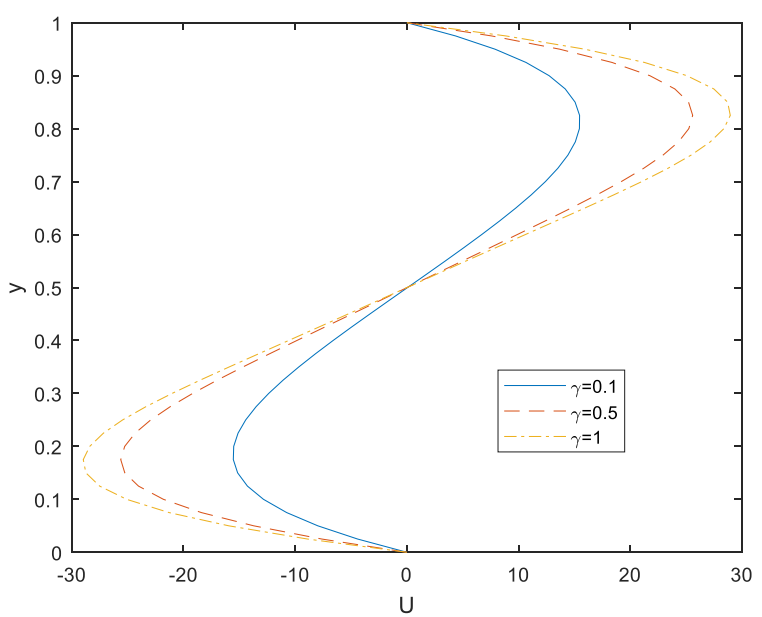

c. $\mathbf{R a}=10^{5}$

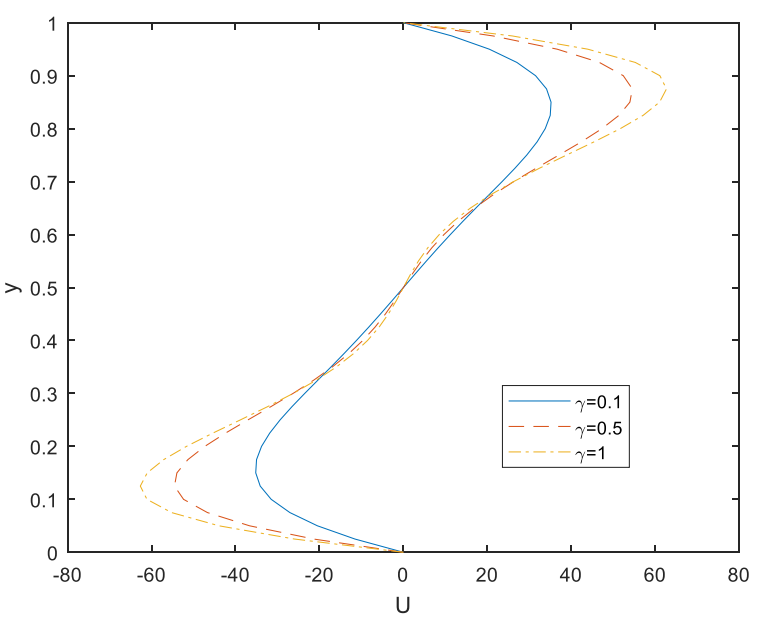

d. $\operatorname{Ra}=10^{6}$

Fig. 7. Variations of non-dimensional velocity $u$ for different values of Casson fluid parameter $(\gamma)$ along the vertical mid-plane for different values of $\operatorname{Ra}$ at $\operatorname{Pr}=6.8$.

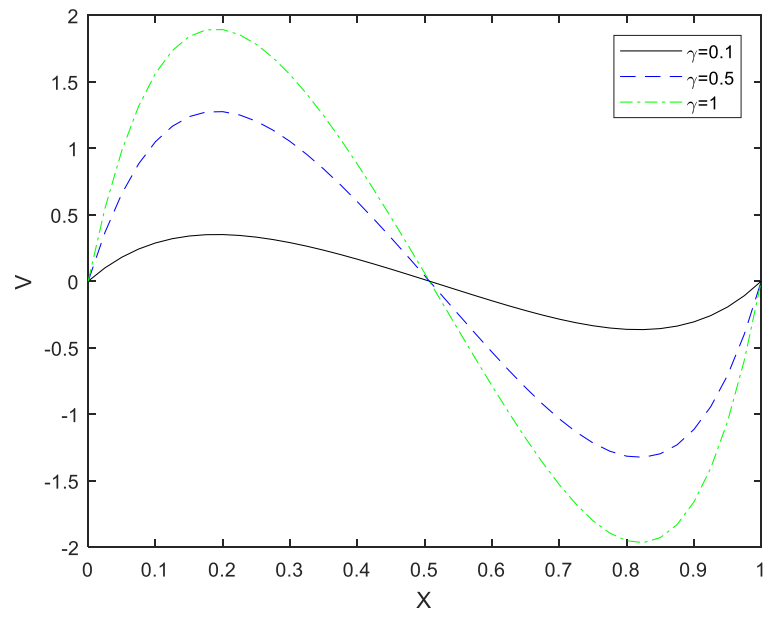

a. $\mathbf{R a}=10^{3}$

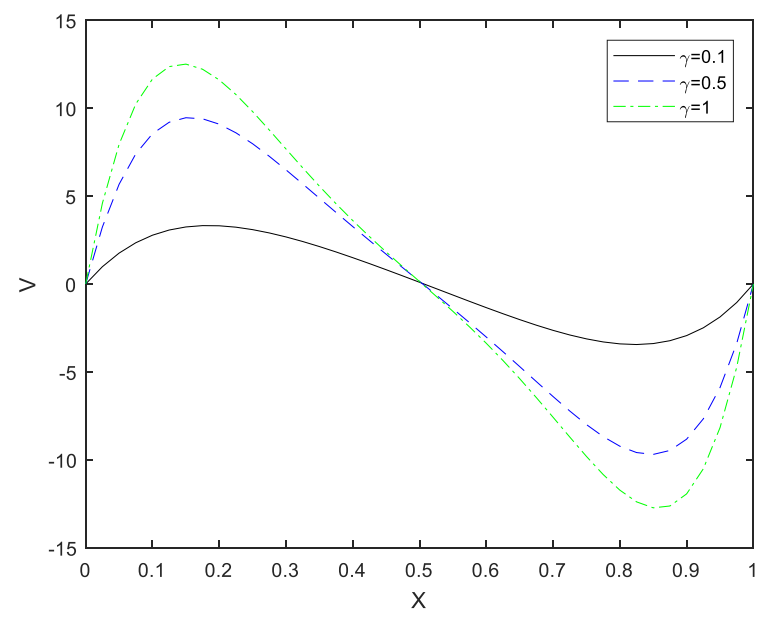

b. $R a=10^{4}$ 


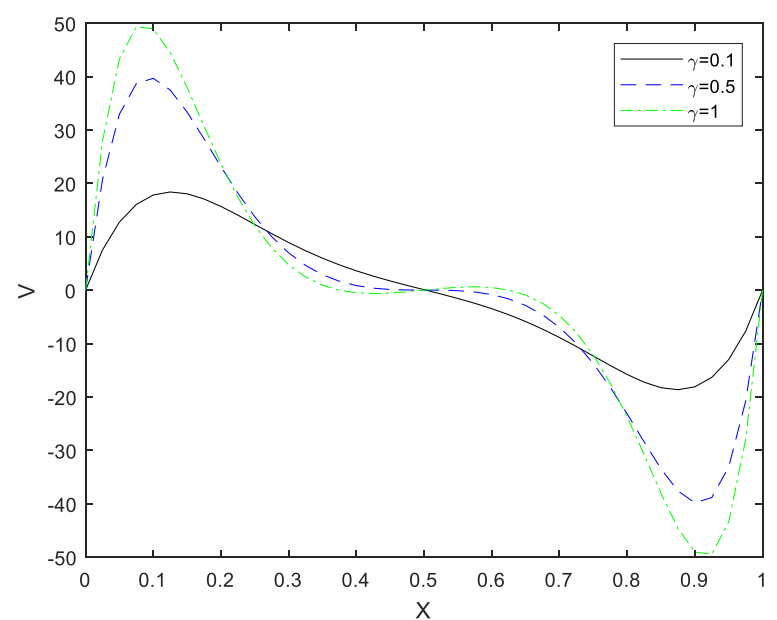

c. $\mathbf{R a}=10^{5}$

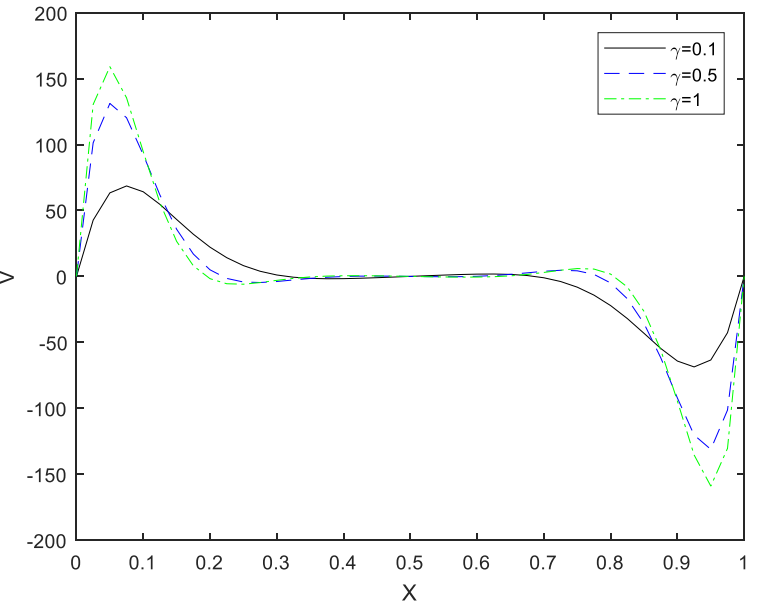

d. $\operatorname{Ra}=10^{6}$

Fig. 8. Variations of non-dimensional velocity $v$ for different values of Casson fluid parameter $(\gamma)$ along the horizontal mid-plane for different values of $\operatorname{Ra}$ at $\operatorname{Pr}=6.8$.

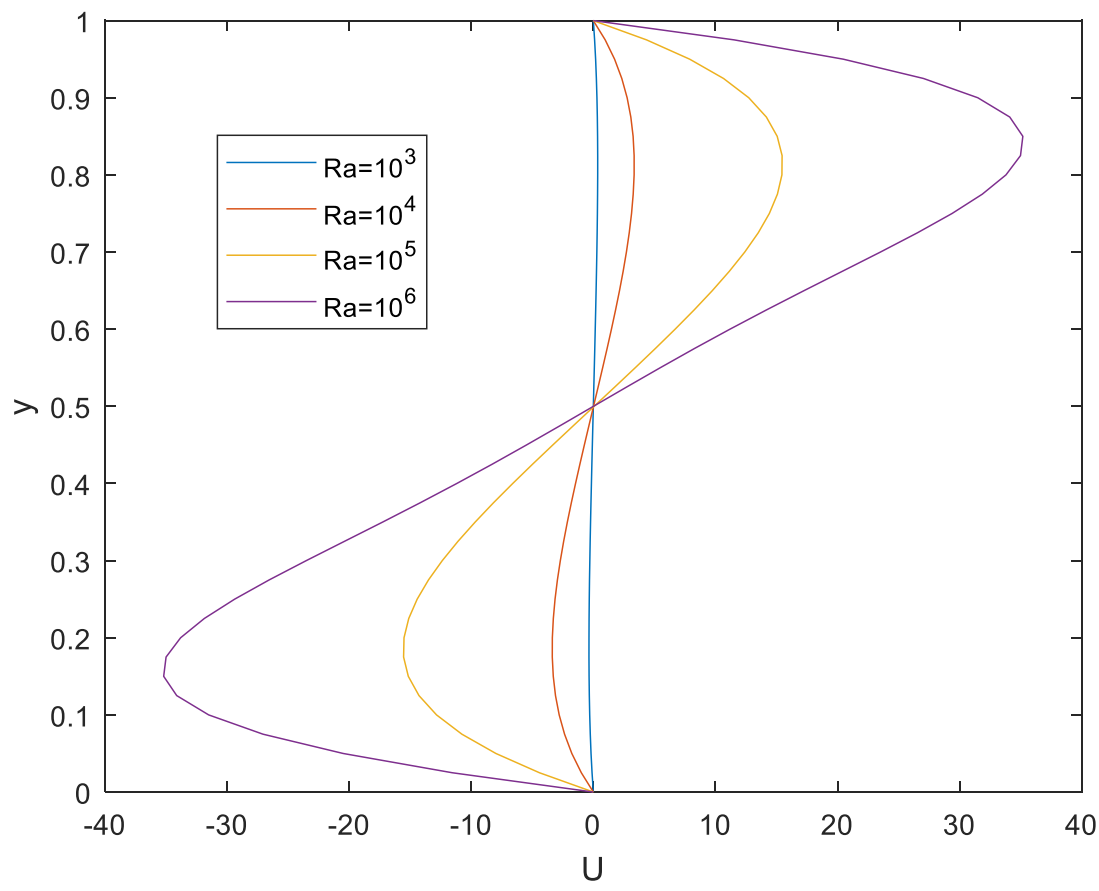

Fig. 9. Variations of non-dimensional velocity $u$ along the vertical mid-plane for different values $\operatorname{Ra}$ at $\operatorname{Pr}=6.8$ and $\gamma=0.1$. 


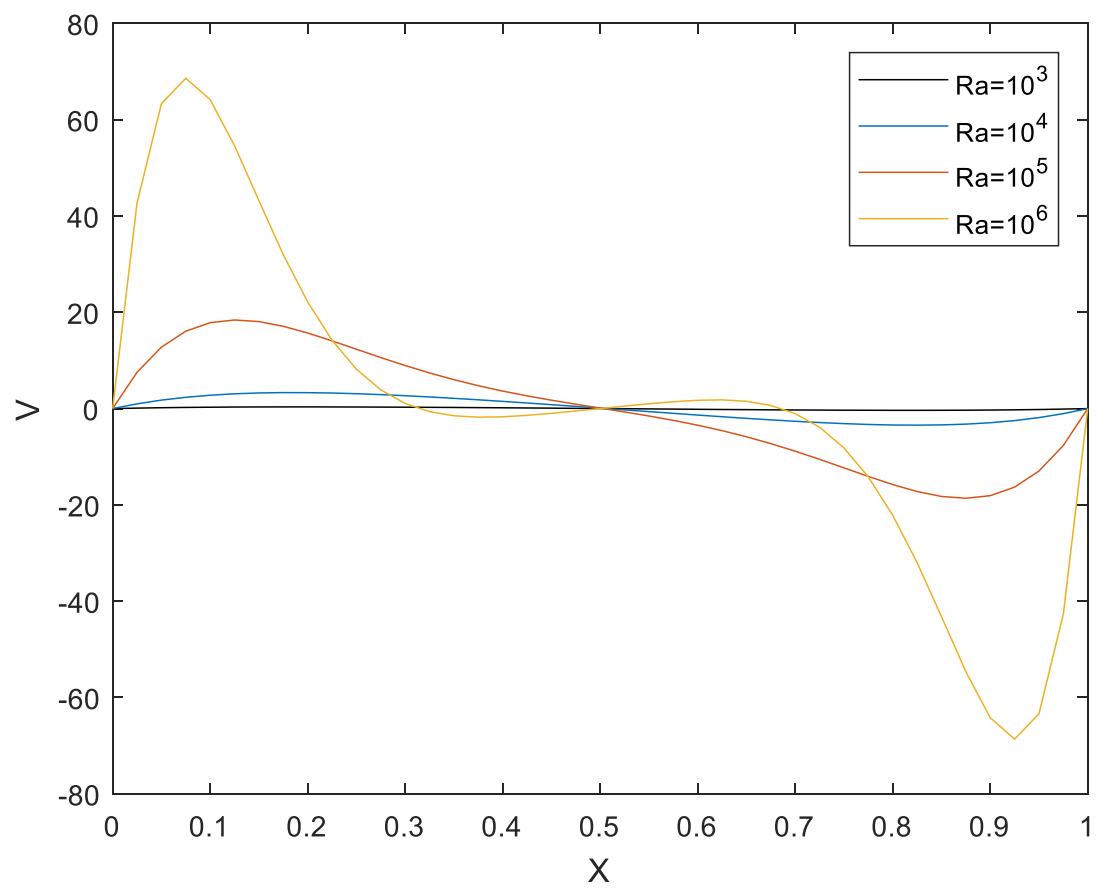

Fig. 10. Variations of non-dimensional velocity $v$ along the horizontal mid-plane for different values $\operatorname{Ra}$ at $\operatorname{Pr}=6.8$ and $\gamma=0.1$.

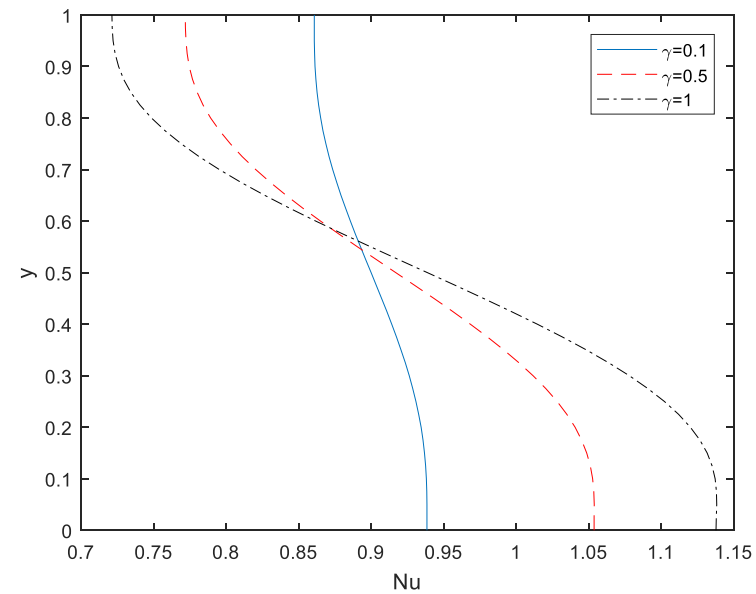

a. $\operatorname{Ra}=10^{3}$

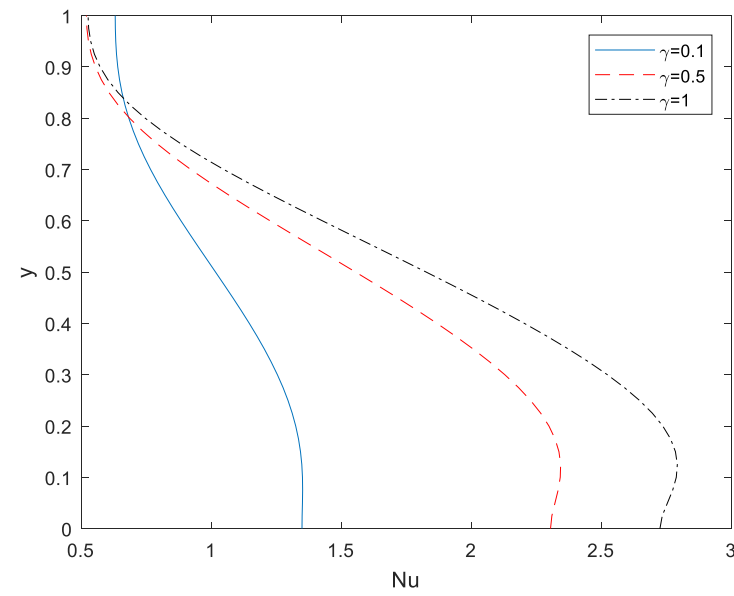

b. $\mathrm{Ra}=10^{4}$ 


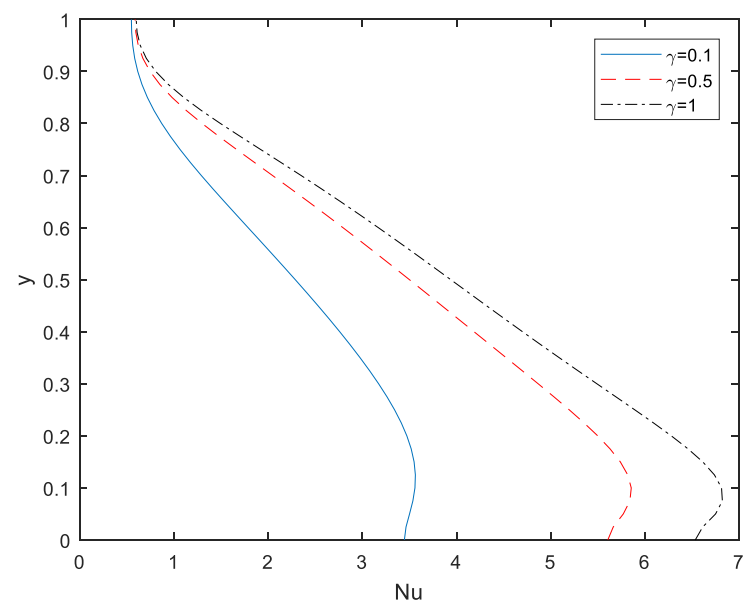

c. $\mathbf{R a}=10^{5}$

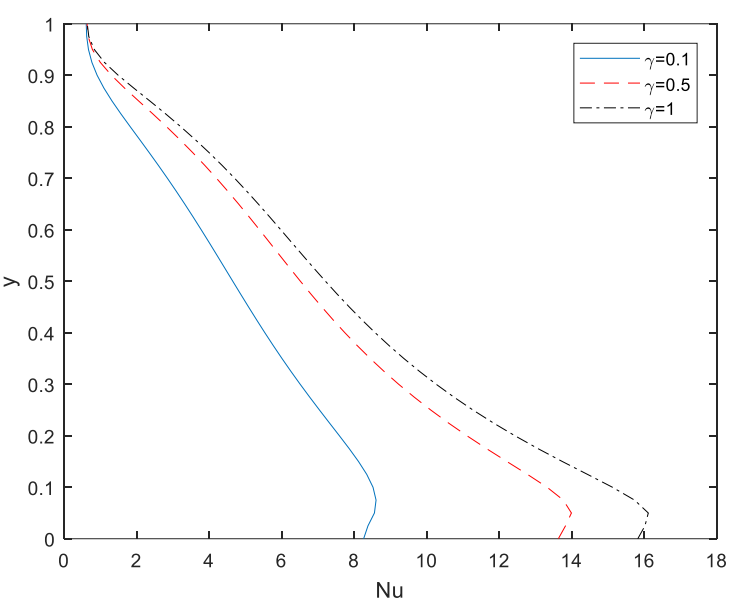

d. $\operatorname{Ra}=10^{6}$

Fig. 11. Variations of local Nusselt number for different values of Casson fluid parameter along the hot wall for different values of $\operatorname{Ra}$ at $\operatorname{Pr}=6.8$.

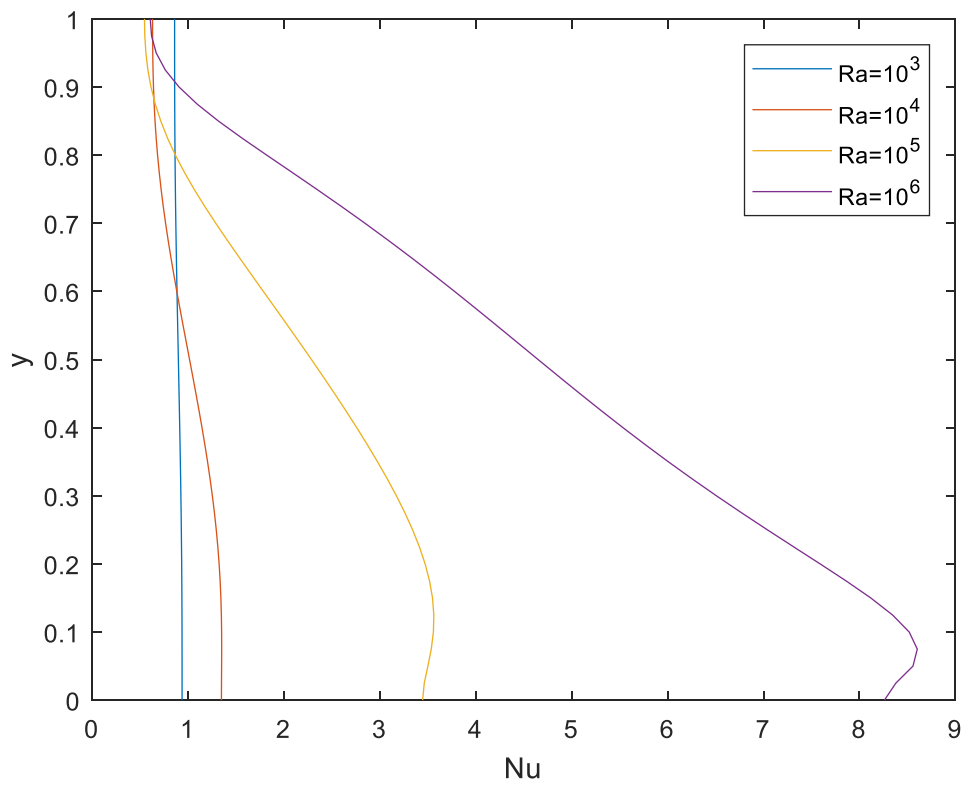

Fig. 12. Variations of local Nusselt number for different values of $\mathrm{Ra}$ along the hot wall at $\mathrm{Pr}$ $=6.8$ and $\gamma=0.1$. 


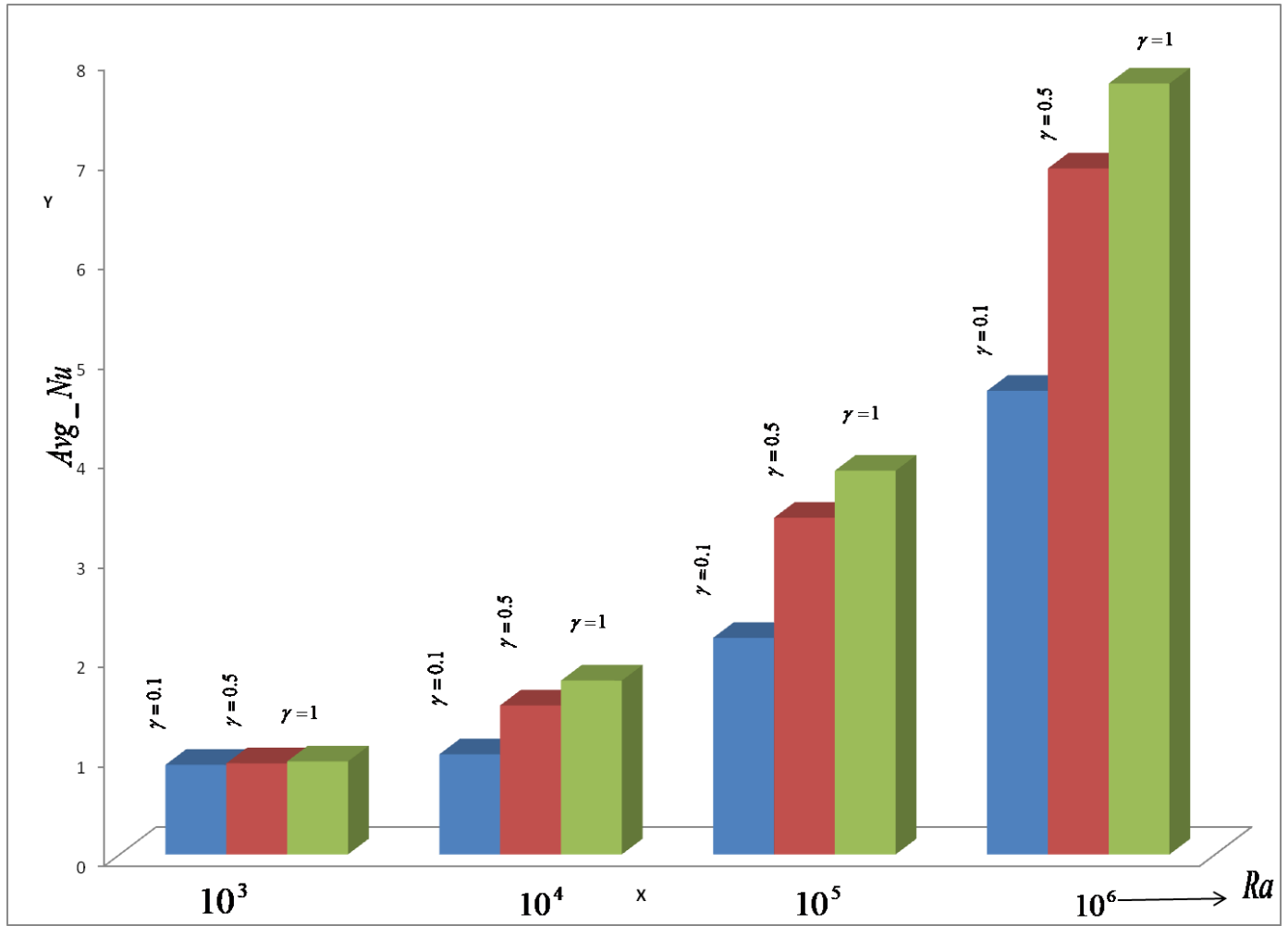

Fig. 13. Effect of Rayleigh number $(R a)$ and Casson parameter $(\gamma)$ on the average Nusselt number for hot wall 